\title{
Dynamics of a non-autonomous incompressible non-Newtonian fluid with delay
}

\author{
Linfang Liu ${ }^{(1),(2)}$, Tomás Caraballo ${ }^{(2), *} \&$ Xianlong $\mathrm{Fu}^{(1)}$ \\ ${ }^{(1)}$ Department of Mathematics, Shanghai Key Laboratory of PMMP, \\ East China Normal University, Shanghai 200241, P.R. China. \\ ${ }^{(2)}$ Dpto. Ecuaciones Diferenciales y Análisis Numérico, \\ Universidad de Sevilla, Apdo. de Correos 1160, 41080-Sevilla, Spain.
}

\begin{abstract}
We first study the well-posedness of a non-autonomous incompressible non-Newtonian fluid with delay. The existence of global solution is obtained by classical Galerkin approximation and the energy method. Actually, we also prove the uniqueness of solution as well as the continuous dependence on the initial value. Then we analyze the long time behavior of the dynamical system associated to the incompressible non-Newtonian fluid. Finally, we establish the existence of pullback attractors for the non-autonomous dynamical system associated to the problem.
\end{abstract}

Key words: Incompressible non-Newtonian equation; pullback attractor; delay; the energy method.

AMS Subject Classification (2010): 35B40, 35Q35, 37L55, 76D03.

\section{Introduction}

As it is well known, the Navier-Stokes model of fluid restricts the linear relation between the stress tensor and the velocity gradient (see [26, 27]). Fluids satisfying such constitutive relationship are called Newtonian fluids, e.g. air, gases, water, motor oil, alcohols, and simply hydrocarbon compounds. However, for many fluid materials, such as molten plastics, synthetic fibers, paints and greases, polymer solutions, suspensions, adhesives, dyes, varnishes, and biological fluids like

${ }^{*}$ Corresponding author. Email: caraball@us.es.

This research was partially supported by the projects MTM2015-63723-P (MINECO/ FEDER, EU) and P12-FQM1492 (Junta de Andalucía), and by NSF of China (Nos. 11671142 and11371087), Science and Technology Commission of Shanghai Municipality (No. 13dz2260400) and Shanghai Leading Academic Discipline Project (No. B407), respectively. 
blood etc., their flow behavior cannot be characterized by Newtonian relationships in the real world. By weaken the constraints of the Stokes hypothesis, the mathematical theory of viscous non-Newtonian fluids generalizes the usual Stokes model in three important aspects: nonlinear constitutive relations between the viscous part of the stress tensor and velocity gradients, dependence of the viscous stress tensor on velocity gradients of order two or higher, and constitutive relations for higher order stress tensors which must be present in the balance of energy equations as soon as higher order velocity gradients are considered into the theory $[3,16]$.

The purpose of this paper is to study the well-posedness and dynamical behavior of the following non-autonomous incompressible non-Newtonian fluid with delay in a 2D bounded domain

$$
\begin{gathered}
\frac{\partial u}{\partial t}+(u \cdot \nabla) u+\nabla p=\nabla \cdot \mu(e(u))+f\left(t, u_{t}\right)+g(x, t), \text { in }(\tau,+\infty) \times \Omega, \\
\nabla \cdot u=0, \text { in }(\tau,+\infty) \times \Omega, \\
u(\tau+\theta, x)=\phi(\theta, x), \quad \theta \in[-h, 0], x \in \Omega .
\end{gathered}
$$

System (1.1)-(1.3) is supplemented by the boundary conditions $\left(v_{i j} e=2 \mu_{1} \frac{\partial e_{i j}}{\partial x_{l}}, i, j, l=1,2\right.$, and $n=\left(n_{1}, n_{2}\right)$ the exterior unit normal to $\left.\partial \Omega\right)$

$$
u=0, \quad v_{i j} n_{j} n_{l}=0, i, j, k=1,2 \text {, on } \partial \Omega \times(\tau,+\infty),
$$

where $\Omega$ is a smooth bounded domain of $\mathbb{R}^{2}$, the unknown vector function $u=u(x, t)=\left(u^{(1)}, u^{(2)}\right)$ denotes the velocity of the fluid, $g(x, t)=g(t)=\left(g^{(1)}, g^{(2)}\right)$ is a time-dependent external function, and the scalar function $p$ represents the pressure. The first condition in (1.4) represents the usual non-slip condition associated with a viscous fluid, while the second one expresses the fact that the first moments of the traction vanish on $\partial \Omega$, it is a direct consequence of the principle of virtual work. The time-dependent delay term $f\left(t, u_{t}\right)$ represents, for instance, the influences of an external force with some kind of delay, memory or hereditary characteristics, although we can also model some kind of feedback controls. Here, $u_{t}$ denotes a segment of the solution, in other words, given $h>0$ and a function $u:[s-h,+\infty) \times \Omega \rightarrow \mathbb{R}^{2}$, for each $t \geq s$ we define the mapping $u_{t}$ : $[-h, 0] \times \Omega \rightarrow \mathbb{R}^{2}$ by

$$
u_{t}(\theta, x)=u(t+\theta, x), \text { for } \theta \in[-h, 0], x \in \Omega .
$$

In this way, this abstract formulation includes several types of delay terms in a unified way. For example, terms like

$$
F_{1}(t, u(t-h)), \quad F_{2}(u(t-\rho(t))), \quad \int_{-h}^{0} F_{3}(t, \theta, u(t+\theta)) d \theta,
$$

where $F_{i}(i=1,2,3)$ are suitable functions, and $\rho: \mathbb{R} \mapsto[0, h]$, can all be described by the following corresponding $f_{i}$ defined as

$$
f_{1}(t, \phi)=F_{1}(t, \phi(-h)), f_{2}(t, \phi)=F_{2}(\phi(-\rho(t))), f_{3}(t, \phi)=\int_{-h}^{0} F_{3}(t, \theta, \phi(\theta)) d \theta,
$$


where $\phi:[-h, 0] \rightarrow X(X$ denotes certain Banach or Hilbert space concerning the spatial variable). Then, when we replace $\phi$ by $u_{t}$ in (1.6), we obtain (1.5). Readers are referred to $[6,10,11]$ for more details.

Problem (1.1)-(1.4) models the motion of an isothermal incompressible viscous fluid with $\mu(e(u))=\left(\mu_{i j}(e(u))\right)_{2 \times 2}$, which is usually called the extra stress tensor of the fluid and is a matrix of order $2 \times 2$ in which

$$
\begin{aligned}
\mu_{i j}(e(u)) & =2 \mu_{0}\left(\epsilon_{0}+|e|^{2}\right)^{-\frac{\alpha}{2}} e_{i j}-2 \mu_{1} \Delta e_{i j}, \quad i, j=1,2, \\
e_{i j} & =e_{i j}(u)=\frac{1}{2}\left(\frac{\partial u_{i}}{\partial x_{j}}+\frac{\partial u_{j}}{\partial x_{i}}\right), \quad|e|^{2}=\sum_{i, j=1}^{2}\left|e_{i j}\right|^{2},
\end{aligned}
$$

where $\mu_{0}, \mu_{1}, \epsilon_{0}$ and $\alpha(0<\alpha<1)$ are positive constants which generally depend on the temperature and pressure. In (1.7) if $\mu_{i j}(e(u))$ depends linearly on $e_{i j}(u)$, then we say the corresponding fluid is a Newtonian one. If the relation between $\mu_{i j}(e(u))$ and $e_{i j}(u)$ is nonlinear, then the fluid is said to be non-Newtonian. One can refer to $[2,3,21,24]$ and related references therein for more physical explanations.

The existence and uniqueness of solution of non-Newtonian flow is studied in $[1,2,3]$, while a maximal compact attractor of a non-Newtonian system in an unbounded channel is obtained in [4]. In [29, 30, 31, 32, 34, 35] the existence of (compact, global, pullback) attractor for a nonNewtonian equation without delay has been analyzed, while [20] focused on pullback attractor of a non-autonomous non-Newtonian equation with variable delays. It is worth mentioning that authors in [33] obtained pullback attractors for a non-Newtonian fluid model with infinite delays, Caraballo and Real [7] proved the existence and uniqueness of solution for functional Navier-Stokes models with delay, and a non-classical non-autonomous diffusion equation with delay was considered in [6].

Enlightened by [6], in this paper we first aim to show the existence, uniqueness and continuity of solutions to (1.1)-(1.4) by the energy method (see $[6,14,15])$ and the classical Galerkin approximation (see [27]). Our second goal is to establish the existence of pullback attractor in space $C\left([-h, 0] ; H^{2}(\Omega)\right)$ by using pullback $\mathcal{D}-\omega$-limit compactness and a priori estimates.

We would like to mention that we will give a relatively complete proof of the existence, uniqueness and continuity of solutions to Eq.(1.1), which will be obtained assuming that $g$ belongs to a more general space than the one in [20], namely, $g \in L_{l o c}^{2}\left(\mathbb{R} ; W^{\prime}\right)$ instead of $g \in L_{l o c}^{2}(\mathbb{R} ; H)$. And the assumption $g \in L_{l o c}^{2}(\mathbb{R} ; H)$ is needed only when we show the existence of pullback absorbing set in the space $C_{W}$. Moreover, we only need $g \in L_{l o c}^{2}(\mathbb{R} ; H)$ and satisfying (4.14), i.e., $\lim _{m \rightarrow+\infty} \sup _{t \geq \tau} \int_{\tau}^{t} e^{-2 \mu_{1} \lambda_{m+1}(t-s)}\|g(s)\|^{2} d s=0$, to establish that the process is pullback $\mathcal{D}-\omega$-limit compact in $C_{W}$. However, in some references, the fact that $g \in C(\mathbb{R} ; H)$ is required to prove the pullback $\mathcal{D}-\omega$-limit compactness in $C_{W}$ which is a much stronger assumption than ours. Besides, in [20] the authors established the existence of pullback attractor for non-Newtonian fluid with variable delay, and we generalize this result to model more general delay. In other words, our result is true for variable and distributed delays. 
The structure of the paper is as follows. In the next section, we first introduce some notations, then recall some definitions and known results concerning pullback attractors for non-autonomous dynamical systems. In Section 3, we prove the existence, uniqueness and continuity of solutions to problem (1.1)-(1.4). Section 4 is devoted to establishing the existence of pullback attractor in space $C\left([-h, 0] ; H^{2}(\Omega)\right)$, while Section 5 provides some conclusions about our work. We also present some technical lemmas that are used in this paper in an Appendix in Section 6.

\section{Preliminaries}

We first recall some notations which are necessary for our analysis although they are similar to those in $[1,3,4,20,34]$, but we prefer to introduce them here for completeness.

$L^{p}(\Omega)$ will denote the $2 \mathrm{D}$ vector Lebesgue space with norm $\|\cdot\|_{L^{p}(\Omega)}$; particularly, $\|\cdot\|_{L^{2}(\Omega)}=\|\cdot\|$,

$H^{m}(\Omega)$ is the $2 \mathrm{D}$ vector Sobolev space $\left\{\phi: \phi=\left(\phi_{1}, \phi_{2}\right) \in L^{2}(\Omega), \nabla^{k} \phi \in L^{2}(\Omega), k \leq m\right\}$ with norm $\|\cdot\|_{H^{m}(\Omega)}$,

$H_{0}^{1}(\Omega)$ is the closure of $\left\{\phi: \phi=\left(\phi_{1}, \phi_{2}\right) \in C^{\infty}(\Omega) \times C^{\infty}(\Omega)\right\}$ in $H^{1}(\Omega)$,

$\mathcal{V}$ denotes the $\left\{\phi \in C^{\infty}(\Omega) \times C^{\infty}(\Omega): \phi=\left(\phi_{1}, \phi_{2}\right), \nabla \cdot \phi=0\right\}$,

$H$ is the closure of $\mathcal{V}$ in $L^{2}(\Omega)$ with norm $\|\cdot\| ; H^{\prime}$ is the dual space of $H$,

$W$ denotes the closure of $\mathcal{V}$ in $H^{2}(\Omega)$ with norm $\|\cdot\|_{W} ; W^{\prime}=$ dual space of $W$,

$(\cdot, \cdot)$-the inner product in $H,\langle\cdot, \cdot\rangle$-the dual pairing between $W$ and $W^{\prime}$.

$\operatorname{dist}_{M}(X, Y)$-the Hausdorff semi-distance between $X, Y \subset M$, where $M$ is a normed space, defined by

$$
\operatorname{dist}_{M}(X, Y)=\sup _{x \in X} \inf _{y \in Y}\|x-y\|_{M}
$$

Set

$$
a(u, v)=\sum_{i, j, k=1}^{2}\left(\frac{\partial e_{i j}(u)}{\partial x_{k}}, \frac{\partial e_{i j}(v)}{\partial x_{k}}\right)=\sum_{i, j, k=1}^{2} \int_{\Omega} \frac{\partial e_{i j}(u)}{\partial x_{k}} \cdot \frac{\partial e_{i j}(v)}{\partial x_{k}} d x, u, v \in W .
$$

On the one hand, from the definition of $a(\cdot, \cdot)$ and Lemma 6.3 in Section 6, we see that $a(\cdot, \cdot)$ defines a positive definite symmetric bilinear form on $W$. As a consequence of the Lax-Milgram Lemma, we obtain an isometric operator $A \in \mathcal{L}\left(W, W^{\prime}\right)$, via

$$
\langle A u, v\rangle=a(u, v), \quad u, v \in W
$$

On the other hand, denoting $D(A)=\{u \in W: A u \in H\}$, it turns out that $D(A)$ is a Hilbert space and $A$ is also an isometry from $D(A)$ to $H$. Actually, $A=P \Delta^{2}$, where $P$ is the Leray projector from $L^{2}(\Omega)$ to $H$ and, for any $u \in D(A)$, we have (see [34] and Appendix for more details)

$$
c_{1}\|u\|_{W} \leq\|A u\| .
$$


We also define a continuous trilinear form on $H_{0}^{1}(\Omega) \times H_{0}^{1}(\Omega) \times H_{0}^{1}(\Omega)$ by

$$
b(u, v, w)=\sum_{i, j=1}^{2} \int_{\Omega} u_{i} \frac{\partial v_{j}}{\partial x_{i}} w_{j} d x, \quad u, v, w \in H_{0}^{1}(\Omega) .
$$

Since $W \subset H_{0}^{1}(\Omega), b(\cdot, \cdot, \cdot)$ is continuous on $W \times W \times W$ and it is easy to check that (see [27])

$$
b(u, v, w)=-b(u, w, v), \quad b(u, v, v)=0, \quad \forall u, v, w \in W .
$$

Now we can define below continuous functional $B(u):=B(u, u)$ from $W \times W$ to $W^{\prime}$, for any $u \in W$, in the following way,

$$
\langle B(u), w\rangle=b(u, u, w), \quad \forall w \in W .
$$

To finish, we set

$$
\mu(u)=2 \mu_{0}\left(\epsilon_{0}+|e(u)|^{2}\right)^{-\alpha / 2},
$$

for $u \in W$, and define $N(u)$ as

$$
\langle N(u), v\rangle=\sum_{i, j=1}^{2} \int_{\Omega} \mu(u) e_{i j}(u) e_{i j}(v) d x, \quad \forall v \in W .
$$

Then the functional $N(u)$ is continuous from $W$ to $W^{\prime}$. When $u \in D(A)$, we can extend $N(u)$ to $H$ by setting

$$
\langle N(u), v\rangle=-\int_{\Omega}\{\nabla \cdot[\mu(u) e(u)] \cdot v\} d x, \forall v \in H .
$$

From a physical point of view, the initial boundary problem of Eq. (1.1) can be formulated as

$$
\begin{gathered}
\frac{\partial u}{\partial t}+(u \cdot \nabla) u+\nabla p=\nabla \cdot\left(2 \mu_{0}\left(\epsilon_{0}+|e|^{2}\right)^{-\frac{\alpha}{2}} e-2 \mu_{1} \Delta e\right)+f\left(t, u_{t}\right)+g(x, t), \text { in }(\tau,+\infty) \times \Omega, \\
\nabla \cdot u=0, \text { in }(\tau,+\infty) \times \Omega, \\
u=0, v_{i j} n_{j} n_{l}=0, \text { on } \partial \Omega \times(\tau, \infty), \\
u(\tau+\theta, x)=\phi(\theta, x), \quad \theta \in[-h, 0], x \in \Omega .
\end{gathered}
$$

As usual, in the variational set-up, we get rid of the pressure and rewrite our problem (2.7)(2.10) in a weak formulation as follows (see $[3,31]$ )

$$
\begin{gathered}
\frac{\partial u}{\partial t}+2 \mu_{1} A u+B(u)+N(u)=f\left(t, u_{t}\right)+g(x, t), \text { in }(\tau,+\infty) \times \Omega, \\
u(\tau+\theta, x)=\phi(\theta, x), \quad \theta \in[-h, 0], x \in \Omega .
\end{gathered}
$$

We now state the assumptions that will be imposed on the function $f:[\tau, T] \times C_{H} \mapsto\left(L^{2}(\Omega)\right)^{2}$ containing the delay along our analysis. We will assume that the given delay term satisfies:

(H1) For any $\xi \in C_{H}$, the mapping $[\tau, T] \ni t \mapsto f(t, \xi) \in\left(L^{2}(\Omega)\right)^{2}$ is measurable, 
(H2) $f(\cdot, 0)=0$,

(H3) $\exists L_{f}>0$ such that for any $t \in[\tau, T]$ and all $\xi, \eta \in C_{H}$,

$$
\|f(t, \xi)-f(t, \eta)\|_{L^{2}(\Omega)} \leq L_{f}\|\xi-\eta\|_{C_{H}},
$$

Remark 2.1. As it is pointed out in $[10,15,25],(H 2)$ is not really a restriction, and condition (H2) and (H3) imply that

$$
\|f(t, \xi)\|_{L^{2}(\Omega)} \leq L_{f}\|\xi\|_{C_{H}}
$$

so that $\|f(\cdot, \xi)\|_{L^{2}(\Omega)} \in L^{\infty}(\tau, T)$.

An example of an operator satisfying assumptions $(\mathrm{H} 1)-(\mathrm{H} 3)$ is given in $[10,15]$.

We now recall some definitions and results concerning dynamical systems and pullback attractors. These definitions and results can be found in [7, 8, 9, 10, 12, 13, 19, 23, 34, 36].

Let $\left(X, d_{X}\right)$ be a metric space, and denote $\mathbb{R}_{d}^{2}=\left\{(t, \tau) \in \mathbb{R}^{2}: \tau \leq t\right\}$. A process $U$ on $X$ is a mapping $\mathbb{R}_{d}^{2} \times X \ni(t, \tau, x) \mapsto U(t, \tau) x \in X$ such that $U(\tau, \tau) x=x$ for any $\tau \in \mathbb{R}, x \in X$, and $U(t, r)(U(r, \tau) x)=U(t, \tau) x$ for any $\tau \leq r \leq t$ and all $x \in X$.

Let $P(X)$ denote the family of all nonempty subsets of $X$, and consider a family of nonempty sets $D_{0}=\left\{D_{0}(t): t \in \mathbb{R}\right\} \subset P(X)$. Let $\mathcal{D}$ be a given nonempty class of sets parameterized in time, $D=\{D(t): t \in \mathbb{R}\} \subset P(X)$. The class $\mathcal{D}$ will be called a universe in $P(X)$.

Definition 2.2. For any $\sigma>0$, we will denote by $\mathcal{D}_{\sigma}(X)$ the class of all families of nonempty subsets $D=\{D(t): t \in \mathbb{R}\} \subset P(X)$ such that

$$
\lim _{t \rightarrow-\infty}\left(e^{\sigma t} \sup _{u \in D(t)}\|u\|_{X}^{2}\right)=0 .
$$

Definition 2.3. It is said that $D_{0}=\left\{D_{0}(t): t \in \mathbb{R}\right\} \subset P(X)$ is pullback $\mathcal{D}$-absorbing for the process $\{U(t, \tau): t \geq \tau\}$ on $X$ if for any $t \in \mathbb{R}$ and any $D=\{D(t): t \in \mathbb{R}\} \in \mathcal{D}$, there exists a $\tau_{0}(t, D) \leq t$ such that

$$
U(t, \tau) D(\tau) \subset D_{0}(t) \text { for all } \tau \leq \tau_{0}(t, D)
$$

Definition 2.4. Let $\{U(t, \tau)\}$ be a process on $X$. We say that $\{U(t, \tau)\}$ is pullback $\mathcal{D}-\omega$-limit compact with respect to each $t \in \mathbb{R}$, if for any family $B=\{B(t): t \in \mathbb{R}\} \in \mathcal{D}$ and for any $\epsilon>0$, there exists $t_{1}=t_{1}(B, t, \epsilon)>0$, such that

$$
\kappa\left(\bigcup_{s \geq t_{1}} U(t, t-s) B(t-s)\right) \leq \epsilon,
$$

where $\kappa$ is the Kuratowski measure of non-compactness (see [23] for more information).

Definition 2.5. The family $\mathcal{A}_{\mathcal{D}}=\left\{\mathcal{A}_{\mathcal{D}}(t): t \in \mathbb{R}\right\} \subset P(X)$ is a pullback $\mathcal{D}$-attractor for the process $\{U(t, \tau): t \geq \tau\}$ in $X$ if : 
(i) for any $t \in \mathbb{R}$, the set $\mathcal{A}_{\mathcal{D}}(t)$ is a nonempty compact subset of $X$,

(ii) $\mathcal{A}_{\mathcal{D}}$ is pullback $\mathcal{D}$-attracting, i.e.,

$$
\lim _{\tau \rightarrow-\infty} \operatorname{dist}_{X}\left(U(t, \tau) D(\tau), \mathcal{A}_{\mathcal{D}}(t)\right)=0, \text { for all } D \in \mathcal{D}, \text { for any } t \in \mathbb{R},
$$

(iii) $\mathcal{A}_{\mathcal{D}}$ is invariant, i.e.,

$$
U(t, \tau) \mathcal{A}_{\mathcal{D}}(\tau)=\mathcal{A}_{\mathcal{D}}(t), \text { for all } \tau \leq t
$$

To analyze our problem with delay, we need to construct our process in a Banach space of segments of solutions. Namely, the space $C_{X}$ which we will define below (see Section 3 for more details). Let $X$ be a Banach space and let $h>0$ be a given positive number (the time delay). Denote by $C_{X}$ the Banach space $C([-h, 0] ; X)$ endowed with the norm $\|\phi\|_{C_{X}}=\sup _{\theta \in[-h, 0]}\|\phi(\theta)\|_{X}$. To study the pullback $\mathcal{D}-\omega$-limit compactness of the process on $C_{X}$, we borrow some techniques from $[19,28]$.

Proposition 2.6. (see [19]) Let $\{U(t, \tau)\}$ be a continuous process on $C_{X}$. Suppose that for each $t \in \mathbb{R}, B=\{B(t): t \in \mathbb{R}\} \in \mathcal{D}$ and $\epsilon>0$, there exist $\tau_{0}=\tau_{0}(t, B, \epsilon)>0$, a finite dimensional subspace $X_{1}$ of $X$ and $\delta>0$ such that

(i) for each fixed $\theta \in[-h, 0]$

$$
\left\|\bigcup_{s \geq \tau_{0}} \bigcup_{u_{t}(\cdot) \in U(t, t-s) B(t-s)} P u(t+\theta)\right\|_{X} \text { is bounded; }
$$

(ii) for all $s \geq \tau_{0}, u_{t}(\cdot) \in U(t, t-s) B(t-s), \theta_{1}, \theta_{2} \in[-h, 0]$ with $\left|\theta_{2}-\theta_{1}\right|<\delta$,

$$
\left\|P\left(u\left(t+\theta_{1}\right)-u\left(t+\theta_{2}\right)\right)\right\|_{X}<\epsilon ;
$$

(iii) for all $s \geq \tau_{0}, u_{t}(\cdot) \in U(t, t-s) B(t-s)$,

$$
\sup _{\theta \in[-h, 0]}\|(I-P) u(t+\theta)\|_{X}<\epsilon,
$$

where $P: X \rightarrow X_{1}$ is the canonical projector. Then $\{U(t, \tau)\}$ is pullback $\mathcal{D}-\omega$-limit compact in $C_{X}$ with respect to each $t \in \mathbb{R}$.

The following proposition is similar to that of $[5,23,36]$.

Proposition 2.7. Let $\{U(t, \tau)\}_{t \geq \tau}$ be a process on Banach space $C_{X}$ and $\mathcal{D}$ be a universe in $P\left(C_{X}\right)$. Then, $\{U(t, \tau)\}_{t \geq \tau}$ possesses a unique pullback $\mathcal{D}$-attractor $\mathcal{A}_{\mathcal{D}}=\left\{\mathcal{A}_{\mathcal{D}}(t): t \in \mathbb{R}\right\}$, for any $t \in \mathbb{R}$ and $D \in \mathcal{D}$,

$$
\mathcal { A } _ { \mathcal { D } } ( t ) = \omega ( D , t ) = \bigcap _ { \tau _ { 0 } \leq t } \longdiv { \bigcup _ { \tau \leq \tau _ { 0 } } U ( t , \tau ) D ( \tau ) }
$$

if and only if

(a) $\{U(t, \tau)\}_{t \geq \tau}$ has a pullback $\mathcal{D}$-absorbing set in $C_{X}$,

(b) $\{U(t, \tau)\}_{t \geq \tau}$ is pullback $\mathcal{D}-\omega$-limit compact in $C_{X}$. 


\section{Existence and continuity of solutions}

In this section, by classical Faedo-Galerkin approximation and the energy method, we prove the existence, uniqueness and continuity of solutions to problem (2.11)-(2.12).

Theorem 3.1. (Existence and uniqueness of solution) Assume (H1)-(H3) hold. Let $g \in L_{\text {loc }}^{2}\left(\mathbb{R}, W^{\prime}\right)$ and $\phi \in C_{H}$. Then, for any $\tau \in \mathbb{R}$,

(a) there exists a unique weak solution u to problem (2.11) satisfying

$$
u \in C([\tau-h, T] ; H) \cap L^{\infty}(\tau, T ; H) \cap L^{2}(\tau, T ; W), \quad \forall T>\tau .
$$

(b) If $\phi \in C_{W}$, and $g \in L_{\text {loc }}^{2}(\mathbb{R}, H)$, then there exists a unique strong solution u to problem (2.11) satisfying

$$
u \in C([\tau-h, T] ; W) \cap L^{\infty}(\tau, T ; W) \cap L^{2}(\tau, T ; D(A)), \quad \forall T>\tau
$$

Proof. We split the proof into several steps.

\section{Step 1. A Galerkin Scheme.}

By the definition of $A$ and the classical spectral theory of elliptic operators (see [24]), we see that operator $A$ possesses a sequence of eigenvalues $\left\{\lambda_{n}\right\}_{n=1}^{\infty}$ and a corresponding family of eigenfunctions $\left\{w^{n}\right\}_{n=1}^{\infty} \subset W \cap D(A)$, which form a basis of $W$ and are orthonormal in $H$, we consider the subspace $W_{m}=\operatorname{span}\left\{w^{1}, w^{2}, \cdots, w^{m}\right\}$, and the projector $P_{m}: H \rightarrow W_{m}$ defined as

$$
P_{m} u=\sum_{n=1}^{m}\left(u, w^{n}\right) w^{n}, \quad u \in H .
$$

Define

$$
u^{m}(t)=\sum_{n=1}^{m} \gamma_{m n} w^{n},
$$

where the upper script $m$ will be used instead of $(m)$ for short, since no confusion is possible with powers of $u$, and the coefficients $\gamma_{m n}$ are required to satisfy the following system:

$$
\begin{gathered}
\left(\frac{\partial}{\partial t} u^{m}(t), w^{n}\right)+2 \mu_{1}\left(A u^{m}, w^{n}\right)+\left\langle B\left(u^{m}(t)\right), w^{n}\right\rangle+\left\langle N\left(u^{m}(t)\right), w^{n}\right\rangle \\
=\left(f\left(t, u_{t}^{m}\right), w^{n}\right)+\left\langle g(t, x), w^{n}\right\rangle, \text { a.e. } t>\tau, \quad 1 \leq n \leq m,
\end{gathered}
$$

and where the equations are understood in the sense of $\mathcal{D}^{\prime}(\tau, T)$, and the initial conditions are

$$
u^{m}(\tau+\theta)=P_{m} \phi(\theta), \text { for } \theta \in[-h, 0] .
$$

The above system of ordinary differential equations with finite delay fulfills the conditions for existence and uniqueness of local solution in [10, Theorem A1, p. 2450]. Hence, we can ensure that problem (3.1) has a unique local solution defined in $\left[\tau, t_{m}\right]$ with $\tau<t_{m} \leq+\infty$ (see [18] for a similar result). 
Next, by a priori estimates, we verify that solutions $u^{m}$ do exist for all time $t \in[\tau,+\infty)$.

\section{Step 2: A priori estimates}

Multiplying (3.1) by $\gamma_{m n}$, summing from $n=1$ to $n=m$, and using Lemma 6.3 in Appendix, we obtain, for all $t \in\left[\tau, t_{m}\right]$, that

$$
\begin{aligned}
& \frac{1}{2} \frac{d}{d t}\left\|u^{m}(t)\right\|^{2}+2 c_{1} \mu_{1}\left\|u^{m}(t)\right\|_{W}^{2}+\left\langle B\left(u^{m}(t)\right), u^{m}(t)\right\rangle+\left\langle N\left(u^{m}(t)\right), u^{m}(t)\right\rangle \\
& \quad \leq\left(f\left(t, u_{t}^{m}\right), u^{m}(t)\right)+\left\langle g, u^{m}(t)\right\rangle .
\end{aligned}
$$

Integrating over $[\tau, t]$,

$$
\begin{aligned}
& \frac{1}{2}\left\|u^{m}(t)\right\|^{2}+2 c_{1} \mu_{1} \int_{\tau}^{t}\left\|u^{m}(s)\right\|_{W}^{2} d s+\int_{\tau}^{t}\left\langle B\left(u^{m}(s)\right), u^{m}(s)\right\rangle d s+\int_{\tau}^{t}\left\langle N\left(u^{m}(s)\right), u^{m}(s)\right\rangle d s \\
& \quad \leq \frac{1}{2}\left\|u^{m}(\tau)\right\|^{2}+\int_{\tau}^{t}\left(f\left(s, u_{s}^{m}\right), u^{m}(s)\right) d s+\int_{\tau}^{t}\left\langle g, u^{m}(s)\right\rangle d s .
\end{aligned}
$$

First, by (2.3) and (2.5),

$$
\int_{\tau}^{t}\left\langle B\left(u^{m}(s)\right), u^{m}(s)\right\rangle d s=0
$$

and

$$
\int_{\tau}^{t}\left\langle N\left(u^{m}(s)\right), u^{m}(s)\right\rangle d s \geq 0
$$

By the fact that $\|v\|_{W} \geq\|v\|$ for all $v \in W$,

$$
\int_{\tau}^{t}\left\langle g, u^{m}(s)\right\rangle d s \leq \frac{c_{1} \mu_{1}}{2} \int_{\tau}^{t}\left\|u^{m}(s)\right\|_{W}^{2} d s+\frac{1}{2 c_{1} \mu_{1}} \int_{\tau}^{t}\|g(s)\|_{W^{\prime}}^{2} d s
$$

From (H3) and Young's inequality,

$$
\begin{aligned}
\int_{\tau}^{t}\left(f\left(s, u_{s}^{m}\right), u^{m}(s)\right) d s & \leq \int_{\tau}^{t}\left\|f\left(s, u_{s}^{m}\right)\right\| \cdot\left\|u^{m}(s)\right\| d s \\
& \leq L_{f} \int_{\tau}^{t}\left\|u_{s}^{m}\right\|_{C_{H}}\left\|u^{m}(s)\right\| d s \\
& \leq \frac{c_{1} \mu_{1}}{2} \int_{\tau}^{t}\left\|u^{m}(s)\right\|_{W}^{2} d s+\frac{L_{f}^{2}}{2 c_{1} \mu_{1}} \int_{\tau}^{t}\left\|u_{s}^{m}\right\|_{C_{H}}^{2} d s .
\end{aligned}
$$

It follows from (3.3)-(3.7) that

$$
\left\|u^{m}(t)\right\|^{2}+2 c_{1} \mu_{1} \int_{\tau}^{t}\left\|u^{m}(s)\right\|_{W}^{2} d s \leq\|\phi\|_{C_{H}}^{2}+\frac{L_{f}^{2}}{c_{1} \mu_{1}} \int_{\tau}^{t}\left\|u_{s}^{m}\right\|_{C_{H}}^{2} d s+\frac{1}{c_{1} \mu_{1}} \int_{\tau}^{t}\|g(s)\|_{W^{\prime}}^{2} d s, \quad \forall t \geq \tau .
$$

Replacing $t$ by $t+\theta$ in (3.8) we obtain

$$
\left\|u_{t}^{m}\right\|_{C_{H}}^{2} \leq\|\phi\|_{C_{H}}^{2}+\frac{L_{f}^{2}}{c_{1} \mu_{1}} \int_{\tau}^{t}\left\|u_{s}^{m}\right\|_{C_{H}}^{2} d s+\frac{1}{c_{1} \mu_{1}} \int_{\tau}^{t}\|g(s)\|_{W^{\prime}}^{2} d s, \quad \forall t \geq \tau,
$$

and therefore, the Gronwall Lemma implies

$$
\left\|u_{t}^{m}\right\|_{C_{H}}^{2} \leq e^{\frac{L_{f}^{2}}{c_{1} \mu_{1}}(t-\tau)}\left(\|\phi\|_{C_{H}}^{2}+\frac{1}{c_{1} \mu_{1}} \int_{\tau}^{t}\|g(s)\|_{W^{\prime}}^{2} d s\right), \quad \forall t \geq \tau, \forall m \geq 1 .
$$


Then, by (3.9), we can check that for each $T>\tau$ and $R>0$, there exists a positive constant $C\left(\tau, T, R, L_{f}\right)$, depending on the constants of the problem $c_{1}, \mu_{1}, L_{f}, g$, and on $\tau, T, R$, such that for all $m \geq 1$,

$$
\left\|u_{t}^{m}\right\|_{C_{H}}^{2}+\left\|u^{m}\right\|_{L^{2}(\tau, T ; W)}^{2} \leq C\left(\tau, T, R, L_{f}\right),\|\phi\|_{C_{H}} \leq R .
$$

In particular, thanks to inequalities (3.8) and (3.9), and the fact that $g \in L_{l o c}^{2}\left(\mathbb{R} ; W^{\prime}\right)$, we deduce

$$
\left\{u^{m}\right\} \text { is bounded in } L^{\infty}(\tau-h, T ; H) \cap L^{2}(\tau, T ; W), \quad \forall T>\tau .
$$

On the other hand, for almost all $t, B(u(t))$ and $N(u(t))$ are elements of $W^{\prime}$, and the measurability of the mappings

$$
t \in[0, T] \rightarrow B(u(t)) \in W^{\prime},
$$

and

$$
t \in[0, T] \rightarrow N(u(t)) \in W^{\prime}
$$

are straightforward. Moreover, thanks to (2.3), the Hölder inequality, embedding theorems, and Lemma 6.1 in Appendix, we have that for all $\varphi \in W$,

$$
\begin{aligned}
|\langle B(u), \varphi\rangle| & =|b(u, u, \varphi)|=|-b(u, \varphi, u)|=\left|\sum_{i, j=1}^{2} \int_{\Omega} u_{i} \frac{\partial \varphi_{j}}{\partial x_{i}} u_{j} d x\right| \\
& \leq c\|u\|_{L^{4}}^{2}\|\varphi\|_{H_{0}^{1}(\Omega)} \leq c\|u\| \cdot\|\nabla u\| \cdot\|\varphi\|_{H_{0}^{1}(\Omega)} \leq c\|u\| \cdot\|\Delta u\| \cdot\|\Delta \varphi\| .
\end{aligned}
$$

Using the fact that $\mu(u)=\mu_{0}\left(\epsilon_{0}+|e|^{2}\right)^{-\frac{\alpha}{2}} \leq \mu_{0} \epsilon_{0}^{-\frac{\alpha}{2}}$, we can also obtain

$$
\begin{aligned}
|\langle N(u), \varphi\rangle| & =\left|2 \sum_{i, j=1}^{2} \int_{\Omega} \mu(e(u)) e_{i j}(u) e_{i j}(\varphi) d x\right| \\
& \leq 2 \mu_{0} \epsilon_{0}^{-\frac{\alpha}{2}} \int_{\Omega} \sum_{i, j=1}^{2}\left|e_{i j}(u) e_{i j}(\varphi)\right| d x \\
& \leq c\|\nabla u\| \cdot\|\nabla \varphi\| \\
& \leq c\|\Delta u\| \cdot\|\Delta \varphi\| .
\end{aligned}
$$

As a consequence of (3.11) and (3.12), the estimates hold true,

$$
\|B(u)\|_{W^{\prime}} \leq c\|u\| \cdot\|\Delta u\|
$$

and

$$
\|N(u)\|_{W^{\prime}} \leq c\|\Delta u\|
$$

Hence,

$$
\int_{\tau}^{T}\|B(u(s))\|_{W^{\prime}}^{2} d s \leq c \int_{\tau}^{T}\|u(s)\|^{2}\|\Delta u(s)\|^{2} d s \leq c \int_{\tau}^{T}\left\|u_{s}\right\|_{C_{H}}^{2}\|\Delta u(s)\|^{2} d s<\infty
$$


and

$$
\int_{\tau}^{T}\|N(u(s))\|_{W^{\prime}}^{2} d s \leq c \int_{\tau}^{T}\|\Delta u(s)\|^{2} d s<\infty .
$$

To this end, we need to show that $\left\{\left(u^{m}\right)^{\prime}\right\}$ is bounded in $L^{2}\left(\tau, T ; W^{\prime}\right)$. Let $\varphi \in C^{1}([0, T], W)$ and $\varphi^{m}$ be the projection of $\varphi$ in $W$, onto the space $W_{m}=\operatorname{span}\left\{w^{1}, w^{2}, \cdots, w^{m}\right\}$. By (3.1), we have

$$
\begin{aligned}
& \int_{\Omega} \frac{\partial u^{m}}{\partial t} w^{n} d x \\
& =-2 \mu_{1} \sum_{i, j, k=1}^{2} \int_{\Omega} \frac{\partial e_{i j}\left(u^{m}\right)}{\partial x_{k}} \frac{\partial e_{i j}\left(w^{n}\right)}{\partial x_{k}} d x-\sum_{i, j=1}^{2} \int_{\Omega} u_{i}^{m} \frac{\partial u_{j}^{m}}{\partial x_{i}} w_{j}^{n} d x \\
& \quad-\sum_{i, j=1}^{2} \int_{\Omega} \mu\left(u^{m}\right) e_{i j}\left(u^{m}\right) e_{i j}\left(w^{n}\right) d x+\int_{\Omega} f\left(t, u_{t}^{m}\right) w^{n} d x+\int_{\Omega} g(x, t) w^{n} d x, n=1,2, \cdots, m .
\end{aligned}
$$

Using (3.17) and the definition of $\varphi^{m}$,

$$
\begin{aligned}
\int_{\tau}^{T} \int_{\Omega} \frac{\partial u^{m}}{\partial t} \varphi d x d t= & \int_{\tau}^{T} \int_{\Omega} \frac{\partial u^{m}}{\partial t} \varphi^{m} d x d t \\
= & -2 \mu_{1} \sum_{i, j, k=1}^{2} \int_{\tau}^{T} \int_{\Omega} \frac{\partial e_{i j}\left(u^{m}\right)}{\partial x_{k}} \frac{\partial e_{i j}\left(\varphi^{m}\right)}{\partial x_{k}} d x d t-\sum_{i, j=1}^{2} \int_{\tau}^{T} \int_{\Omega} u_{i}^{m} \frac{\partial u_{j}^{m}}{\partial x_{i}} \varphi_{j}^{m} d x d t \\
& -\sum_{i, j=1}^{2} \int_{\tau}^{T} \int_{\Omega} \mu\left(u^{m}\right) e_{i j}\left(u^{m}\right) e_{i j}\left(\varphi^{m}\right) d x d t+\int_{\tau}^{T} \int_{\Omega} f\left(t, u_{t}^{m}\right) \varphi^{m} d x d t \\
& +\int_{\tau}^{T} \int_{\Omega} g(x, t) \varphi^{m} d x d t \\
= & I_{1}+I_{2}+I_{3}+I_{4}+I_{5} .
\end{aligned}
$$

From (3.10),

$$
\left|I_{1}+I_{4}+I_{5}\right| \leq C_{1}\left\|\varphi^{m}\right\|_{L^{2}(\tau, T ; W)}
$$

By a similar argument to that one in (3.11) and (3.12), we can check that

$$
\left|I_{2}\right| \leq C_{2} \int_{\tau}^{T}\left\|u^{m}\right\| \cdot\left\|\nabla u^{m}\right\| \cdot\left\|\nabla \varphi^{m}\right\| d t \leq C_{3}\left\|\varphi^{m}\right\|_{L^{2}(\tau, T ; W)}
$$

as well as

$$
\left|I_{3}\right| \leq C_{4}\left\|\varphi^{m}\right\|_{L^{2}(\tau, T ; W)}
$$

Hence, from (3.17)-(3.21), we can conclude that

$$
\left|\int_{\tau}^{T} \int_{\Omega} \frac{\partial u^{m}}{\partial t} \varphi d x d t\right| \leq C_{5}\left\|\varphi^{m}\right\|_{L^{2}(\tau, T ; W)} \leq C_{5}\|\varphi\|_{L^{2}(\tau, T ; W)},
$$

and

$$
\left\|\frac{\partial u^{m}}{\partial t}\right\|_{L^{2}\left(\tau, T ; W^{\prime}\right)} \leq C_{6},
$$


where $C_{i}(i=1,2, \cdots, 6)$ are positive constants. Thus,

$$
\left\{\left(u^{m}\right)^{\prime}\right\} \text { is bounded in } L^{2}\left(\tau, T ; W^{\prime}\right), \quad \forall T \geq \tau \text {. }
$$

\section{Step 3: The energy method and compactness results}

Now, we combine some well-known compactness results with the energy method to pass to the limit in a subsequence of $\left\{u^{m}\right\}$ to obtain a solution of (1.1). Observe that

$$
\left.u^{m}\right|_{[\tau-h, \tau]}=P_{m} \phi \rightarrow \phi \text { in } C_{H} .
$$

By Step 1, Step 2 and compactness theorem, we deduce that there exist a subsequence (which we relabel the same) $\left\{u^{m}\right\}$, a function $u \in C([\tau-h, \infty) ; H)$, with $\left.u\right|_{[\tau-h, \tau]}=\phi, u \in L^{2}(\tau, T ; W)$, $\chi \in L^{2}\left(\tau, T ; W^{\prime}\right)$ for all $T>\tau$, and an element $\xi \in L^{\infty}(\tau, T, H)$ for all $T>\tau$, such that

$$
\begin{aligned}
& u^{m} \stackrel{*}{\rightarrow} u \text { weakly-star in } L^{\infty}(\tau, T ; H), \\
& u^{m} \rightarrow u \text { weakly in } L^{2}(\tau, T ; W), \\
& \left(u^{m}\right)^{\prime} \rightarrow \chi \text { weakly in } L^{2}\left(\tau, T ; W^{\prime}\right), \\
& u^{m} \rightarrow u \text { strongly in } L^{2}(\tau, T ; H), \\
& f\left(\cdot, u^{m}\right) \stackrel{*}{\rightarrow} \xi \text { weakly-star in } L^{\infty}(\tau, T ; H) .
\end{aligned}
$$

We first prove that $\chi=u^{\prime}=\frac{d u}{d t}$. Indeed, since the approximate solutions $\left\{u^{m}\right\}$ satisfy

$$
u^{m}(s)=P_{m} \phi(\tau)+\int_{\tau}^{s} \frac{d u^{m}}{d t} d t, \quad s \in[\tau, T], m=1,2, \cdots .
$$

From (3.25), we know

$$
P_{m} \phi(\tau) \rightarrow \phi(\tau)
$$

Then

$$
u(s)=\phi(\tau)+\int_{\tau}^{s} \chi d t
$$

by [27, Lemma 3.1, Chapter II], we immediately deduce that $\chi=u^{\prime}=\frac{d u}{d t}$.

Using (3.26) $)_{4}$, we can also assume that

$$
u^{m}(t) \rightarrow u(t) \text { in } H \text { a.e. } t \in[\tau, T]
$$

which is not enough to deduce that $\xi(\cdot)=f(\cdot, u$. .

However, we can obtain convergence for all $t>\tau$ with a little more effort and in a more general case. Notice that,

$$
u^{m}(t)-u^{m}(s)=\int_{s}^{t}\left(u^{m}\right)^{\prime}(r) d r \text { in } W^{\prime}, \forall s, t \in[\tau, T]
$$


and by (3.24) we have that $\left\{u^{m}\right\}$ is equi-continuous on $[\tau, T]$ with values in $W^{\prime}$, for all $T>\tau$.

Since the injection of $W$ in $H$ is compact, the injection of $H$ into $W^{\prime}$ is compact as well. Thus, from (3.10) and the equi-continuity of $\left\{u^{m}\right\}$ in $W^{\prime}$, using Arzelà-Ascoli theorem, we have (again, up to a subsequence)

$$
u^{m} \rightarrow u \text { in } C\left([\tau, T] ; W^{\prime}\right), \quad \forall T>\tau .
$$

This, jointly with the fact $H \subset W^{\prime}$, (3.10) and [27, Lemma 3.3, Chapter II], allows us to claim that for any sequence $\left\{t_{m}\right\} \subset[\tau, \infty)$, with $t_{m} \rightarrow t$,

$$
u^{m}\left(t_{m}\right) \rightarrow u(t) \text { weakly in } H,
$$

where we have used (3.28) in order to identify which is the weak limit.

Now we prove that in fact

$$
u^{m}\left(t_{m}\right) \rightarrow u(t) \text { in } C([\tau, T] ; H) \forall T>\tau .
$$

If not, then, taking into account that $u \in C([\tau, \infty) ; H)$, there would exist $T>\tau, \epsilon_{1}>0$, a value $t_{0} \in[\tau, T]$, and subsequences (relabelled the same) $\left\{u^{m}\right\}$ and $\left\{t_{m}\right\} \subset[\tau, T]$, with $\lim _{m \rightarrow+\infty} t_{m}=t_{0}$, such that

$$
\left\|u^{m}\left(t_{m}\right)-u\left(t_{0}\right)\right\| \geq \epsilon_{1}, \forall m \geq 1 .
$$

To conclude that this is false, we use an energy method. Note that the following energy equality holds for all $u^{m}$ :

$$
\begin{aligned}
& \frac{1}{2} \frac{d}{d t}\left\|u^{m}(t)\right\|^{2}+2 \mu_{1} a\left(u^{m}(t), u^{m}(t)\right)+\left\langle B\left(u^{m}(t)\right), u^{m}(t)\right\rangle+\left\langle N\left(u^{m}(t)\right), u^{m}(t)\right\rangle \\
& \quad=\left(f\left(t, u_{t}^{m}\right), u^{m}(t)\right)+\left\langle g, u^{m}(t)\right\rangle .
\end{aligned}
$$

By Lemma 6.3, we find that

$$
\begin{aligned}
& \frac{1}{2} \frac{d}{d t}\left\|u^{m}(t)\right\|^{2}+2 c_{1} \mu_{1}\left\|u^{m}(t)\right\|_{W}^{2}+\left\langle B\left(u^{m}(t)\right), u^{m}(t)\right\rangle+\left\langle N\left(u^{m}(t)\right), u^{m}(t)\right\rangle \\
& \quad \leq\left(f\left(t, u_{t}^{m}\right), u^{m}(t)\right)+\left\langle g, u^{m}(t)\right\rangle .
\end{aligned}
$$

Integrating (3.32) over $[s, t]$ with respect to $t$,

$$
\begin{aligned}
& \frac{1}{2}\left\|u^{m}(t)\right\|^{2}+2 c_{1} \mu_{1} \int_{s}^{t}\left\|u^{m}(r)\right\|_{W}^{2} d r+\int_{s}^{t}\left\langle B\left(u^{m}(r)\right), u^{m}(r)\right\rangle d r+\int_{s}^{t}\left\langle N\left(u^{m}(r)\right), u^{m}(r)\right\rangle d r \\
& \leq \frac{1}{2}\left\|u^{m}(s)\right\|^{2}+\int_{s}^{t}\left(f\left(r, u_{r}^{m}\right), u^{m}(r)\right) d r+\int_{s}^{t}\left\langle g(r), u^{m}(r)\right\rangle d r .
\end{aligned}
$$

Since $\left\langle B\left(u^{m}(r)\right), u^{m}(r)\right\rangle=0$ and $\left\langle N\left(u^{m}(r)\right), u^{m}(r)\right\rangle \geq 0$, and

$$
\begin{aligned}
\int_{s}^{t}\left(f\left(r, u_{r}^{m}\right), u^{m}(r)\right) d r & \leq \frac{c_{1} \mu_{1}}{2} \int_{s}^{t}\left\|u^{m}(r)\right\|_{W}^{2} d r+\frac{1}{2 c_{1} \mu_{1}} \int_{s}^{t}\left\|f\left(r, u_{r}^{m}\right)\right\|^{2} d r \\
& \leq \frac{c_{1} \mu_{1}}{2} \int_{s}^{t}\left\|u^{m}(r)\right\|_{W}^{2} d r+\frac{L_{f}^{2}}{2 c_{1} \mu_{1}} \int_{s}^{t}\left\|u_{r}^{m}\right\|_{C_{H}} d r \\
& \leq \frac{c_{1} \mu_{1}}{2} \int_{s}^{t}\left\|u^{m}(r)\right\|_{W}^{2} d r+C(t-s), \forall \tau \leq s \leq t \leq T,
\end{aligned}
$$


where $C=\frac{L_{f}^{2} D}{2 c_{1} \mu_{1}}$, and $D$ corresponds to the upper bound of $\left\|u_{t}\right\|_{C_{H}}$, it follows

$$
\left\|u^{m}(t)\right\|^{2} \leq\left\|u^{m}(s)\right\|^{2}+2 \int_{s}^{t}\left\langle g(r), u^{m}(r)\right\rangle d r+2 C(t-s), \quad \forall \tau \leq s \leq t \leq T .
$$

On the one hand, observe that by (3.26), passing to the limit in (3.1), we have that $u \in C([\tau, T] ; H)$ is a solution of a similar problem to (1.1), namely,

$$
\left(\frac{\partial}{\partial t} u(t), w\right)+2 \mu_{1} a(u(t), w)+\langle B(u(t)), w\rangle+\langle N(u(t)), w\rangle=(\xi, w)+\langle g, w\rangle, \quad \forall w \in W,
$$

fulfilled with the initial datum $u(\tau)=\phi(0)$. Therefore, it satisfies the energy equality

$$
\begin{aligned}
& \frac{1}{2}\|u(t)\|^{2}+2 \mu_{1} \int_{s}^{t} a(u(r), u(r)) d r+\int_{s}^{t}\langle B(u(r)), u(r)\rangle d r+\int_{s}^{t}\langle N(u(r)), u(r)\rangle d r \\
& =\frac{1}{2}\|u(s)\|^{2}+\int_{s}^{t}(\xi(r), u(r)) d r+\int_{s}^{t}\langle g(r), u(r)\rangle d r, \quad \forall \tau \leq s \leq t \leq T .
\end{aligned}
$$

On the other hand, from $(3.26)_{5}$ we deduce that

$$
\int_{s}^{t}\|\xi(r)\|^{2} d r \leq \lim _{m \rightarrow+\infty} \inf \int_{s}^{t}\left\|f\left(r, u_{r}^{m}\right)\right\|^{2} d r \leq D(t-s), \quad \forall \tau \leq s \leq t \leq T
$$

which implies that $u$ also satisfies inequality (3.34) (here we applied Lemma 6.3) with the same constant $c_{1}$.

Now, consider the functions $J_{m}, J:[\tau, T] \mapsto \mathbb{R}$ defined by

$$
\begin{aligned}
& J_{m}(t)=\frac{1}{2}\left\|u^{m}(t)\right\|^{2}-\int_{\tau}^{t}\left\langle g(r), u^{m}(r)\right\rangle d r-C(t-\tau), \\
& J(t)=\frac{1}{2}\|u(t)\|^{2}-\int_{\tau}^{t}\langle g(r), u(r)\rangle d r-C(t-\tau),
\end{aligned}
$$

with $C$ defined in (3.34). By (3.34) and the analogous inequality for $u$, it is clear that $J_{m}$ and $J$ are non-increasing continuous functions. Moreover, by (3.26) and (3.27),

$$
J_{m}(t) \rightarrow J(t) \text { a.e. } t \in[\tau, T]
$$

Now we are ready to prove that

$$
u^{m}\left(t_{m}\right) \rightarrow u\left(t_{0}\right) \text { in } H
$$

Recall that from (3.29) we have

$$
\left\|u\left(t_{0}\right)\right\| \leq \lim _{m \rightarrow+\infty} \inf \left\|u^{m}\left(t_{m}\right)\right\|
$$

Therefore, if we show that

$$
\lim _{m \rightarrow+\infty} \sup \left\|u^{m}\left(t_{m}\right)\right\| \leq\left\|u\left(t_{0}\right)\right\|,
$$

then combining with (3.37), we can obtain $\lim _{m \rightarrow+\infty}\left\|u^{m}\left(t_{m}\right)\right\|=\left\|u\left(t_{0}\right)\right\|$, which means (3.36) holds true. 
Note that the case $t_{0}=\tau$ follows directly from (3.25) and (3.34) with $s=\tau$. Hence, we can assume that $t_{0}>\tau$. Owing to this result, we approach $t_{0}$ from the left by a sequence $\left\{\tilde{t}_{k}\right\}$, namely, $\lim _{k \rightarrow+\infty} \tilde{t}_{k} \nearrow t_{0}$, being $\left\{\tilde{t}_{k}\right\}$ values where (3.35) holds. Since $J(\cdot)$ is continuous at $t_{0}$, for any $\epsilon>0$ there is $k_{\epsilon}$ such that $\left|J\left(\tilde{t}_{k}\right)-J\left(t_{0}\right)\right|<\frac{\epsilon}{2}$ for all $k \geq k_{\epsilon}$. On the other hand, taking $m \geq m\left(k_{\epsilon}\right)$ such that $t_{m}>\tilde{t}_{k_{\epsilon}}$, as $J_{m}$ is non-increasing and for all $\tilde{t}_{k}$ the convergence (3.35) holds, one has

$$
J_{m}\left(t_{m}\right)-J\left(t_{0}\right) \leq\left|J_{m}\left(\tilde{t}_{k_{\epsilon}}\right)-J\left(\tilde{t}_{k_{\epsilon}}\right)\right|+\left|J\left(\tilde{t}_{k_{\epsilon}}\right)-J\left(t_{0}\right)\right|
$$

and taking $m \geq m^{\prime}\left(k_{\epsilon}\right) \geq m\left(k_{\epsilon}\right)$, such that $\left|J_{m}\left(\tilde{t}_{k_{\epsilon}}\right)-J\left(\tilde{t}_{k_{\epsilon}}\right)\right|<\frac{\epsilon}{2}$. It can also be deduced from (3.26) that

$$
\int_{\tau}^{t_{m}}\left\langle g(r), u^{m}(r)\right\rangle d r \rightarrow \int_{\tau}^{t_{0}}\langle g(r), u(r)\rangle d r
$$

We conclude that (3.38) holds. Thus, (3.36) and finally (3.30) are also true, as claimed. This also implies, thanks to (3.25), that $u_{t}^{m} \rightarrow u_{t}$ in $C_{H}$ for all $t \geq \tau$. Therefore, we identify the weak limit $\xi$ from (3.26). Indeed, from the above convergence and since $f$ satisfies (H3), we have that $f\left(\cdot, u_{.}^{m}\right) \rightarrow f(\cdot, u$. $)$ in $L^{2}\left(\tau, T ;\left(L^{2}(\Omega)\right)^{2}\right)$ for all $T>\tau$. Thus, we can pass to the limit finally in (3.1) concluding that $u$ solves (1.1).

\section{Step 4: The uniqueness of solution}

This can be obtained in the following way. Consider two weak solutions of (1.1), $u$ and $v$, with the same initial data, and denote $w=u-v$. We notice that

$$
\begin{gathered}
|b(u, v, w)| \leq 2^{-\frac{1}{2}}\|u\|^{\frac{1}{2}}\|\nabla u\|^{\frac{1}{2}}\|\nabla v \mid\| w\left\|^{\frac{1}{2}}\right\| \nabla w \|^{\frac{1}{2}}, \quad u, v, w \in W . \\
\frac{\partial w}{\partial t}+2 \mu_{1} A w+B(u)-B(v)+N(u)-N(v)=f\left(t, u_{t}\right)-f\left(t, v_{t}\right),
\end{gathered}
$$

with initial value

$$
w(\tau)=0 .
$$

Take the inner product of (3.39) with $w$ to yield that

$$
\frac{1}{2} \frac{d}{d t}\|w\|^{2}+2 \mu_{1} a(w, w)+\langle B(u)-B(v), w\rangle+\langle N(u)-N(v), w\rangle=\left(f\left(t, u_{t}\right)-f\left(t, v_{t}\right), w\right) .
$$

From the monotonicity of $\mu(u)$, it follows that

$$
\langle N(u)-N(v), w\rangle=2 \int_{\Omega}\left[\mu(u) e_{i j}(u)-\mu(v) e_{i j}(v)\right] e_{i j}(w) d x \geq 0,
$$

and we also have

$$
\langle B(u)-B(v), w\rangle=b(u, u, w)-b(v, v, w)=b(w, v, w) .
$$

Then, for some $\alpha_{i}>0, i=1,2,3$,

$$
\begin{aligned}
|b(u, u, w)-b(v, v, w)| & \leq\left|\int_{\Omega} w_{i} \frac{\partial u_{j}}{\partial x_{i}} w_{j} d x\right| \leq \alpha_{1}\|w\|_{L^{4}}^{2}\|\nabla u\| \\
& \leq \alpha_{2}\|w\|_{L^{2}}\|\nabla w\| \cdot\|\nabla u\| \leq \alpha_{3}\|w\|_{L^{2}}\|\Delta w\| \cdot\|\Delta u\| .
\end{aligned}
$$


Using Lemma 6.3, together with (3.42)-(3.43), we find that, for some $c>0$,

$$
\begin{aligned}
\frac{d}{d t}\|w\|^{2} & \leq c\|w\| \cdot\|\Delta w\| \cdot\|\Delta u\|+2\left(f\left(t, u_{t}\right)-f\left(t, v_{t}\right), w\right) \\
& \leq c\|w\| \cdot\|\Delta w\| \cdot\|\Delta u\|+2 L_{f}\left\|u_{t}-v_{t}\right\| \cdot\|w\| \\
& \leq c\|w\| \cdot\|\Delta w\| \cdot\|\Delta u\|+2 L_{f}\left\|w_{t}\right\| \cdot\|w\| \\
& \leq c_{1} \mu_{1}\|w\|_{W}^{2}+c\|w\|^{2}\|\Delta u\|^{2}+2 L_{f}\left\|w_{t}\right\|_{C_{H}}^{2} \text {, for all } t>\tau,
\end{aligned}
$$

and therefore,

$$
\frac{d}{d t}\|w\|^{2} \leq c\|w\|^{2}\|\Delta u\|^{2}+2 L_{f}\left\|w_{t}\right\|_{C_{H}}^{2}, \text { for all } t>\tau
$$

Integrating the above inequality over $[\tau, t]$ with respect to $t$,

$$
\|w(t)\|^{2} \leq c \int_{\tau}^{t}\|w(r)\|^{2}\|\Delta u(r)\|^{2} d r+2 L_{f} \int_{\tau}^{t}\left\|w_{r}\right\|_{C_{H}}^{2} d r .
$$

Hence,

$$
\left\|w_{t}\right\|_{C_{H}}^{2} \leq \int_{\tau}^{t}\left(c\|\Delta u(r)\|^{2}+2 L_{f}\right)\left\|w_{r}\right\|_{C_{H}}^{2} d r
$$

By the Gronwall lemma, we have

$$
\left\|w_{t}\right\|_{C_{H}}^{2} \equiv 0
$$

Finally, the regularity in $(b)$ is a consequence of well-known regularity results and the fact that, if $g \in L_{l o c}^{2}\left(\mathbb{R} ;\left(L^{2}(\Omega)\right)^{2}\right)$, then the function $\hat{g}(t)=g(t)+f\left(t, u_{t}\right), t>\tau$, belongs to $L_{l o c}^{2}\left(\tau, \infty ;\left(L^{2}(\Omega)\right)^{2}\right)$.

The proof is finished.

Theorem 3.2. (Continuous dependence of solutions on initial values) Let $g \in L_{\text {loc }}^{2}\left(\mathbb{R} ; W^{\prime}\right), f$ : $\mathbb{R} \times C_{H} \mapsto\left(L^{2}(\Omega)\right)^{2}$ satisfying $(H 1)-(H 3)$, and $\phi, \psi \in C_{H}$ be given. Let us denote $u=u(\cdot ; \tau, \phi)$ and $v=v(\cdot ; \tau, \psi)$ the corresponding weak solutions to problem (1.1). Then, the following estimate holds:

$$
\left\|u_{t}-v_{t}\right\|_{C_{H}}^{2} \leq\|\phi-\psi\|_{C_{H}}^{2} \exp \left\{\int_{\tau}^{t}\left(c\|\Delta u(s)\|^{2}+2 L_{f}\right) d s\right\} .
$$

Proof. Denote $w=u-v$. Analogously to the arguments in Theorem 3.1 for the proof of uniqueness of weak solution to problem (1.1) we obtain

$$
\frac{\partial w}{\partial t}+2 \mu_{1} A w+(B(u)-B(v))+(N(u)-N(v))=f\left(t, u_{t}\right)-f\left(t, v_{t}\right) .
$$

Multiplying (3.44) by $w$,

$$
\frac{d}{d t}\|w\|^{2}+3 c_{1} \mu_{1}\|w\|_{W}^{2} \leq c\|w\|^{2}\|\Delta u\|^{2}+2 L_{f}\left\|w_{t}\right\|_{C_{H}}^{2} .
$$


Integrate the above inequality over $[\tau, t]$ with respect to $t$ to get

$$
\|w(t)\|^{2} \leq\|w(\tau)\|^{2}+c \int_{\tau}^{t}\|w(s)\|^{2}\|\Delta u(s)\|^{2} d s+2 L_{f} \int_{\tau}^{t}\left\|w_{s}\right\|_{C_{H}}^{2} d s,
$$

particularly,

$$
\left\|w_{t}\right\|_{C_{H}}^{2} \leq\|w(\tau)\|^{2}+\int_{\tau}^{t}\left(c\|\Delta u(s)\|^{2}+2 L_{f}\right)\left\|w_{s}\right\|_{C_{H}}^{2} d s .
$$

Again by the Gronwall lemma, we have

$$
\left\|w_{t}\right\|_{C_{H}}^{2} \leq\|w(\tau)\|^{2} \exp \left\{\int_{\tau}^{t}\left(c\|\Delta u(s)\|^{2}+2 L_{f}\right) d s\right\}
$$

namely,

$$
\left\|u_{t}-v_{t}\right\|_{C_{H}}^{2} \leq\|\phi-\psi\|_{C_{H}}^{2} \exp \left\{\int_{\tau}^{t}\left(c\|\Delta u(s)\|^{2}+2 L_{f}\right) d s\right\} .
$$

The proof is completed immediately.

\section{Existence of pullback $\mathcal{D}$-attractor}

In this section, we analyze the existence of pullback $\mathcal{D}$-attractor in $C_{W}$.

\subsection{Uniform estimates}

Now, by the previous results, we are able to define correctly a process $U$ on $C_{H}$ and $C_{W}$ associated to (2.11), and then to obtain the existence of pullback attractors.

Theorem 4.1. Let $g \in L_{l o c}^{2}\left(\mathbb{R} ; W^{\prime}\right)$ and $f: \mathbb{R} \times C_{H} \rightarrow\left(L^{2}(\Omega)\right)^{2}$ satisfying $(H 1)-(H 3)$. Then, the process $U(t, \tau): C_{H} \rightarrow C_{H}$, with $\tau \leq t$, given by

$$
U(t, \tau) \phi=u_{t}(\cdot ; \tau, \phi)
$$

where $u=u(\cdot ; \tau, \phi)$ is the unique weak solution to (2.11), defines a continuous process on $C_{H}$.

Proof. It is a consequence of Theorem 3.1 and 3.2.

Remark 4.2. By a reasoning similar to the one in Theorem 3.2, we can conclude that $U$ depends continuously on the initial values in $C_{W}$, which jointly with Theorem 3.1, allow us to show that $U$ is also a well-defined process on $C_{W}$ with $g \in L_{l o c}^{2}(\mathbb{R} ; H)$ and initial datum $\phi \in C_{W}$.

Next, we show the existence of pullback $\mathcal{D}$-absorbing sets of $U$ in $C_{H}$ and $C_{W}$, and then verify the pullback $\mathcal{D}-\omega$-limit compactness of $U$ in $C_{W}$. Hereafter, we suppose that

$$
\text { there exists } 0<\beta<c_{1} \mu_{1} \text { such that } \sigma:=\beta-\frac{L_{f}^{2} e^{\beta h}}{c_{1} \mu_{1}}>0 \text {, }
$$


and

$$
\int_{-\infty}^{t} e^{\sigma s}\left(\|g(s)\|_{W^{\prime}}^{2}+\|g(s)\|^{2}\right) d s<\infty, \forall t \in \mathbb{R}
$$

and denote by $\mathcal{D}$ the class of all families of nonempty subsets $D=\{D(t)\}_{t \in \mathbb{R}} \subset P\left(C_{X}\right)$ such that

$$
\lim _{t \rightarrow-\infty}\left(e^{\sigma t} \sup _{u \in D(t)}\|u\|_{C_{X}}^{2}\right)=0,
$$

where $\sigma$ is defined in (4.1), $C_{X}=C_{H}$ or $C_{X}=C_{W}$.

Remark 4.3. If $c_{1} \mu_{1} h>1$, there exists $\beta$ which satisfies (4.1) when $e h L_{f}^{2}<c_{1} \mu_{1}$; if $c_{1} \mu_{1} h \leq 1$, then we can choose $\beta$ which satisfies (4.1) as long as $e^{c_{1} \mu_{1} h} L_{f}^{2}<\left(c_{1} \mu_{1}\right)^{2}$ holds.

We now prove the existence of a pullback absorbing set in $C_{H}$.

Lemma 4.4. (Pullback absorbing set in $C_{H}$ ) Assume that (H1)-(H3) hold and $g \in L_{l o c}^{2}\left(\mathbb{R} ; W^{\prime}\right)$. Let $B=\{B(t): t \in \mathbb{R}\} \in \mathcal{D}$. Then, there exists $T_{B}>0$, such that for any $t \in \mathbb{R}$, all $r>T_{B}$ and $\phi \in B(t-r) \subset C_{H}$, the weak solution $u(\cdot ; t-r, \phi)$ of $E q$. (2.11) satisfies

$$
\left\|u_{t}\right\|_{C_{H}}^{2}=\|U(t, t-r) \phi\|_{C_{H}}^{2} \leq \rho_{1}^{2}(t)
$$

where $\rho_{1}^{2}(t):=1+e^{\beta h-\sigma t} \int_{-\infty}^{t} e^{\sigma s}\|g(s)\|_{W^{\prime}}^{2} d s$.

Proof. The uniform estimates that we require for the solutions which define the process $U$ are analogous to those provided in the proof of theorems 3.1-3.2, but there with Galerkin approximations.

For the sake of brevity, we only sketch the main ideas:

Multiplying (2.11) by $u$, by Lemma 6.3, we have

$$
\frac{1}{2} \frac{d}{d t}\|u\|^{2}+2 c_{1} \mu_{1}\|u\|_{W}^{2}+\langle B(u), u\rangle+\langle N(u), u\rangle \leq\left(f\left(t, u_{t}\right), u\right)+\langle g, u\rangle .
$$

Observe that

$$
\langle B(u), u\rangle=0,\langle N(u), u\rangle \geq 0 .
$$

By (H3) and the Young inequality,

$$
\left(f\left(t, u_{t}\right), u\right) \leq\left\|f\left(t, u_{t}\right)\right\| \cdot\|u\| \leq L_{f}\left\|u_{t}\right\| \cdot\|u\| \leq \frac{L_{f}^{2}}{2 c_{1} \mu_{1}}\left\|u_{t}\right\|_{C_{H}}^{2}+\frac{c_{1} \mu_{1}}{2}\|u\|_{W}^{2},
$$

and

$$
\langle g, u\rangle \leq \frac{c_{1} \mu_{1}}{2}\|u\|_{W}^{2}+\frac{1}{2 c_{1} \mu_{1}}\|g\|_{W^{\prime}}^{2}
$$

From the above inequalities we obtain

$$
\frac{d}{d t}\|u\|^{2}+2 c_{1} \mu_{1}\|u\|_{W}^{2} \leq \frac{L_{f}^{2}}{c_{1} \mu_{1}}\left\|u_{t}\right\|_{C_{H}}^{2}+\frac{1}{c_{1} \mu_{1}}\|g\|_{W^{\prime}}^{2} .
$$


Multiplying (4.3) by $e^{\beta t}$ with $0<\beta<c_{1} \mu_{1}$, and integrating the resulting over $[\tau, t]$ yield

$$
e^{\beta t}\|u(t)\|^{2} \leq e^{\beta \tau}\|u(\tau)\|^{2}+\frac{L_{f}^{2}}{c_{1} \mu_{1}} \int_{\tau}^{t} e^{\beta s}\left\|u_{s}\right\|_{C_{H}}^{2} d s+\frac{1}{c_{1} \mu_{1}} \int_{\tau}^{t} e^{\beta s}\|g(s)\|_{W^{\prime}}^{2} d s .
$$

In particular we have

$$
e^{\beta t}\left\|u_{t}\right\|_{C_{H}}^{2} \leq e^{\beta(\tau+h)}\|u(\tau)\|^{2}+\frac{L_{f}^{2} e^{\beta h}}{c_{1} \mu_{1}} \int_{\tau}^{t} e^{\beta s}\left\|u_{s}\right\|_{C_{H}}^{2} d s+\frac{e^{\beta h}}{c_{1} \mu_{1}} \int_{\tau}^{t} e^{\beta s}\|g(s)\|_{W^{\prime}}^{2} d s .
$$

By Lemma 6.4 in Appendix, we obtain that

$$
e^{\beta t}\left\|u_{t}\right\|_{C_{H}}^{2} \leq e^{\beta(h+\tau)+\frac{L_{f}^{2} f^{\beta h}}{c_{1} \mu_{1}}(t-\tau)}\|\phi\|_{C_{H}}^{2}+\frac{e^{\beta h}}{c_{1} \mu_{1}} \int_{\tau}^{t} e^{\beta s+\frac{L_{f}^{2} \beta^{\beta h}}{c_{1} \mu_{1}}(t-s)}\|g(s)\|_{W^{\prime}}^{2} d s, \forall t \geq \tau,
$$

which means that

$$
\left\|u_{t}\right\|_{C_{H}}^{2} \leq e^{\beta h-\sigma(t-\tau)}\|\phi\|_{C_{H}}^{2}+\frac{e^{\beta h}}{c_{1} \mu_{1}} \int_{\tau}^{t} e^{-\sigma(t-s)}\|g(s)\|_{W^{\prime}}^{2} d s, \forall t \geq \tau .
$$

We now consider the initial time $t-r$ instead of $\tau$, and then

$$
\begin{aligned}
\left\|u_{t}\right\|_{C_{H}}^{2}=\|U(t, t-r) \phi\|_{C_{H}}^{2} & \leq e^{\beta h-\sigma r}\|\phi\|_{C_{H}}^{2}+e^{\beta h-\sigma t} \int_{t-r}^{t} e^{\sigma s}\|g(s)\|_{W^{\prime}}^{2} d s \\
& \leq e^{\beta h-\sigma r}\|\phi\|_{C_{H}}^{2}+e^{\beta h-\sigma t} \int_{-\infty}^{t} e^{\sigma s}\|g(s)\|_{W^{\prime}}^{2} d s .
\end{aligned}
$$

We deduce from (4.5) that there exists $T_{B}>0$, such that for all $r>T_{B}$ and all $t \in \mathbb{R}$, it holds

$$
\left\|u_{t}\right\|_{C_{H}}^{2} \leq 1+e^{\beta h-\sigma t} \int_{-\infty}^{t} e^{\sigma s}\|g(s)\|_{W^{\prime}}^{2} d s:=\rho_{1}^{2}(t)
$$

The proof is finished.

Denoting by $B_{C_{H}}\left(0, \rho_{1}(t)\right)$ the closed ball in $C_{H}$ of center zero and radius $\rho_{1}(t)$, it is easy to check that $\lim _{t \rightarrow-\infty} e^{\sigma t} \rho_{1}^{2}(t)=0$. Hence, $B_{C_{H}}\left(0, \rho_{1}(t)\right)$ is a pullback $\mathcal{D}$-absorbing set for the process $U$ in $C_{H}$.

To our purpose, the following lemma is needed.

Lemma 4.5. Assume that (H1)-(H3) hold and $g \in L_{\text {loc }}^{2}\left(\mathbb{R} ; W^{\prime}\right)$. Then for $T_{B}$ the absorbing time corresponding to the set $B_{C_{H}}\left(0, \rho_{1}(t)\right)$ in Lemma 4.4, there holds

$$
\int_{t-1}^{t} a(u(s ; t-r, \phi), u(s ; t-r, \phi)) d s \leq \rho_{2}^{2}(t),
$$

for all $r \geq T_{B}, t \in \mathbb{R}$, where $\rho_{2}^{2}(t):=c \rho_{1}^{2}(t)+c e^{-\sigma(t-1)} \int_{-\infty}^{t} e^{\sigma s}\|g(s)\|_{W^{\prime}}^{2} d s$. 
Proof. Denote $u(\cdot)=u\left(\cdot ; t_{0}-r, \phi\right)$ for $\phi \in B\left(t_{0}-r\right) \subset C_{H}$, where $t_{0} \in \mathbb{R}$ is a fixed, but arbitrary, number, and let us take $r \geq T_{B}$, where we have chosen the same $\sigma$ than in that proof. We can then integrate (4.3) over $[t-1, t]$ for $t \geq t_{0}$ and $r \geq T_{B}$,

$$
2 c_{1} \mu_{1} \int_{t-1}^{t}\|u(s)\|_{W}^{2} d s \leq\|u(t-1)\|^{2}+\frac{L_{f}^{2}}{c_{1} \mu_{1}} \int_{t-1}^{t}\left\|u_{s}\right\|_{C_{H}}^{2} d s+\frac{1}{c_{1} \mu_{1}} \int_{t-1}^{t}\|g(s)\|_{W^{\prime}}^{2} d s .
$$

Therefore,

$$
\begin{aligned}
\frac{c_{1} \mu_{1}}{c_{2}} \int_{t-1}^{t} a(u(s), u(s)) d s & \leq c_{1} \mu_{1} \int_{t-1}^{t}\|u(s)\|_{W}^{2} d s \\
& \leq \frac{1}{2}\|u(t-1)\|^{2}+\frac{L_{f}^{2}}{2 c_{1} \mu_{1}} \int_{t-1}^{t}\left\|u_{s}\right\|_{C_{H}}^{2} d s+\frac{1}{2 c_{1} \mu_{1}} \int_{t-1}^{t}\|g(s)\|_{W^{\prime}}^{2} d s .
\end{aligned}
$$

Notice that by Lemma 4.4, for all $r \geq T_{B}$, it follows

$$
\frac{1}{2}\|u(t-1)\|^{2}+\frac{L_{f}^{2}}{2 c_{1} \mu_{1}} \int_{t-1}^{t}\left\|u_{s}\right\|_{C_{H}}^{2} d s \leq c \rho_{1}^{2}(t)
$$

and

$$
\int_{t-1}^{t}\|g(s)\|_{W^{\prime}}^{2} d s \leq \int_{t-1}^{t} e^{\sigma(s-t+1)}\|g(s)\|_{W^{\prime}}^{2} d s \leq e^{-\sigma(t-1)} \int_{-\infty}^{t} e^{\sigma s}\|g(s)\|_{W^{\prime}}^{2} d s .
$$

Hence, we can deduce for all $r \geq T_{B}$,

$$
\int_{t-1}^{t} a(u(s), u(s)) d s \leq c \rho_{1}^{2}(t)+c e^{-\sigma(t-1)} \int_{-\infty}^{t} e^{\sigma s}\|g(s)\|_{W^{\prime}}^{2} d s:=\rho_{2}^{2}(t) .
$$

The proof is completed immediately.

Lemma 4.6. (Pullback absorbing set in $C_{W}$ ) Assume that (H1)-(H3) hold and $g \in L_{l o c}^{2}(\mathbb{R} ; H)$. Then the weak solution $u$ of (2.11) satisfies

$$
\left\|u_{t}\right\|_{C_{W}}^{2}=\|U(t, t-r) \phi\|_{C_{W}}^{2} \leq \rho_{3}^{2}(t)
$$

for all $t \geq T_{B}+1+h$ and $t \in \mathbb{R}$, where $\rho_{3}^{2}(t):=\frac{1}{c_{1}}\left(a_{2}+a_{3}\right) e^{a_{1}}, a_{1}=c\left(1+\rho_{1}^{2}(t) \rho_{2}^{2}(t)\right), a_{2}=$ $c\left(\rho_{1}^{2}(t)+e^{-\sigma(t-1)} \int_{-\infty}^{t} e^{\sigma s}\|g(s)\|^{2} d s\right)$, and $a_{3}=\rho_{2}^{2}(t)$.

Proof. Denote $u(\cdot)=u\left(\cdot ; t_{0}-r, \phi\right)$ for $\phi \in C_{W}$, where $t_{0} \in \mathbb{R}$ is a fixed number, and let us take $r \geq T_{B}$. We multiply (2.11) by $A u$ and obtain that for $s \geq t_{0}$,

$$
\frac{1}{2} \frac{d}{d s} a(u(s), u(s))+2 \mu_{1}\|A u\|^{2}+\langle B(u), A u\rangle+\langle N(u), A u\rangle=\left(f\left(s, u_{s}\right), A u\right)+(g, A u) .
$$

On the one hand,

$$
\left(f\left(s, u_{s}\right), A u\right) \leq\left\|f\left(s, u_{s}\right)\right\| \cdot\|A u\| \leq L_{f}\left\|u_{s}\right\|_{C_{H}} \cdot\|A u\| \leq \frac{\mu_{1}}{4}\|A u\|^{2}+\frac{L_{f}^{2}}{\mu_{1}}\left\|u_{s}\right\|_{C_{H}}^{2},
$$




$$
(g, A u) \leq \frac{\mu_{1}}{4}\|A u\|^{2}+\frac{1}{\mu_{1}}\|g\|^{2} .
$$

By Hölder's inequality and the Gagliardo-Nirenberg inequality,

$$
\begin{aligned}
|\langle B(u), A u\rangle| \leq\|B u\| \cdot\|A u\| & \leq\|u\|_{L^{4}}\|\nabla u\|_{L^{4}} \cdot\|A u\| \leq c\|u\|_{W^{\frac{1}{2}}}^{\frac{1}{2}}\|u\|_{H^{1}}\|u\|^{\frac{1}{2}}\|A u\| \\
& \leq c\|A u\|^{\frac{3}{2}}\|u\|_{H^{1}}\|u\|^{\frac{1}{2}} \leq \frac{\mu_{1}}{4}\|A u\|^{2}+\frac{1}{\mu_{1}}\|u\|^{2}\|u\|_{H^{1}}^{4} .
\end{aligned}
$$

Moreover, from the definition of $N(u)$, one can check that

$$
\begin{aligned}
\langle N(u), A u\rangle & =-\int_{\Omega}\{\nabla \cdot[\mu(u) \cdot e(u)]\} \cdot A u d x \\
& \leq c(\|\nabla u\|+\|\Delta u\|) \cdot\|A u\| \leq \frac{\mu_{1}}{4}\|A u\|^{2}+c\|\Delta u\|^{2} .
\end{aligned}
$$

It follows from (4.7)-(4.11) that

$$
\frac{d}{d s} a(u, u)+2 \mu_{1}\|A u\|^{2} \leq \frac{2 L_{f}^{2}}{\mu_{1}}\left\|u_{s}\right\|_{C_{H}}^{2}+\frac{2}{\mu_{1}}\|g\|^{2}+c\left(1+a(u, u)\|u\|^{2}\right) a(u, u) .
$$

On the other hand, from Lemma 4.4 we have for all $r \geq T_{B}$,

$$
\int_{t-1}^{t}\left[\frac{2 L_{f}^{2}}{\mu_{1}}\left\|u_{s}\right\|_{C_{H}}^{2}+\frac{2}{\mu_{1}}\|g\|^{2}\right] d s \leq c\left(\rho_{1}^{2}(t)+e^{-\sigma(t-1)} \int_{-\infty}^{t} e^{\sigma s}\|g(s)\|^{2} d s\right) .
$$

In view of Lemma 4.5, for all $r \geq T_{B}$,

$$
\int_{t-1}^{t} a(u(s), u(s))\|u(s)\|^{2} d s \leq \int_{t-1}^{t} a(u(s), u(s)) e^{-\sigma s} e^{\sigma s}\left\|u_{s}\right\|_{C_{H}}^{2} d s \leq e^{\sigma} \rho_{1}^{2}(t) \rho_{2}^{2}(t) .
$$

Now, by Lemma 6.3 and 6.5 in Appendix, we can conclude that

$$
\|u(s)\|_{W}^{2} \leq \frac{1}{c_{1}} a(u, u) \leq \frac{1}{c_{1}}\left(a_{2}+a_{3}\right) e^{a_{1}}, \text { for all } s \geq t_{0}+1, \text { provided } r \geq T_{B},
$$

where $a_{1}=c\left(1+\rho_{1}^{2}(t) \rho_{2}^{2}(t)\right), a_{2}=c\left(\rho_{1}^{2}(t)+e^{-\sigma(t-1)} \int_{-\infty}^{t} e^{\sigma s}\|g(s)\|^{2} d s\right), a_{3}=\rho_{2}^{2}(t)$, and consequently, if we take $r \geq T_{B}+h+1$,

$$
\sup _{\theta \in[-h, 0]}\left\|u\left(t_{0}+\theta\right)\right\|_{W}^{2} \leq \frac{1}{c_{1}}\left(a_{2}+a_{3}\right) e^{a_{1}}:=\rho_{3}^{2}(t),
$$

where the constants $a_{1}, a_{2}, a_{3}$ and $c_{1}$ in (4.13) are independent of the fixed time $t_{0} \in \mathbb{R}$. Thus (4.13) holds true for all $t_{0} \in \mathbb{R}$. Denoting from now on

$$
u(\cdot)=u(\cdot ; t-r, \phi)
$$

we have for all $t \in \mathbb{R}, r \geq T_{B}+h+1$,

$$
\left\|u_{t}\right\|_{C_{W}}^{2}=\|U(t, t-r) \phi\|_{C_{W}}^{2} \leq \frac{1}{c_{1}}\left(a_{2}+a_{3}\right) e^{a_{1}}:=\rho_{3}^{2}(t),
$$

as claimed. 
Obviously, it is easy to check that $\lim _{t \rightarrow-\infty} e^{\sigma t} \rho_{3}^{2}(t)=0$. Denote by $B_{C_{W}}\left(0, \rho_{3}(t)\right)$ the closed ball in $C_{W}$ of center zero and radius $\rho_{3}(t)$. Thus, $B_{C_{W}}\left(0, \rho_{3}(t)\right)$ is a pullback $\mathcal{D}$-absorbing set for the process $U$ in $C_{W}$.

From now on, we assume that

$$
\lim _{m \rightarrow+\infty} \sup _{t \geq \tau} \int_{\tau}^{t} e^{-2 \mu_{1} \lambda_{m+1}(t-s)}\|g(s)\|^{2} d s=0 .
$$

Remark 4.7. An example for $g$ satisfying (4.14) is given in [28], i.e., if $g$ is normal in $L_{l o c}^{2}(\mathbb{R} ; H)$, then (4.14) holds, which is proved in Lemma 3.1 of [22].

Now, we are in a position to prove pullback $\mathcal{D}-\omega$-limit compactness of the process $U$ in $C_{W}$.

Lemma 4.8. Suppose that (H1)-(H3) and (4.14) hold. Then the process $\{U(t, \tau)\}$ corresponding to problem (2.11)-(2.12) is pullback $\mathcal{D}-\omega$-limit compact on $C_{W}$.

Proof. By the classical spectral theory of elliptic operators, there exists a sequence $\left\{\lambda_{n}\right\}_{n=1}^{\infty}$ satisfying

$$
0<\lambda_{1} \leq \lambda_{2} \leq \cdots \leq \lambda_{n} \leq \cdots, \lambda_{n} \rightarrow+\infty \text { as } n \rightarrow+\infty,
$$

and a family of elements $\left\{w_{n}\right\}_{n=1}^{\infty} \subset D(A)$, which forms a basis of $W$ and is orthonormal in $H$, such that

$$
A w_{n}=\lambda_{n} w_{n}, \quad \forall n \in \mathbb{N} .
$$

Let $W_{m}=\operatorname{span}\left\{w_{1}, w_{2}, \cdots, w_{m}\right\}$, where $m \in \mathbb{N}$ will be specified later. Then $W_{m}$ is a finitedimensional subspace of $W$. Denote by $P_{m}$ the orthogonal projector from $W$ into $W_{m}$ and we obviously have $\left\|P_{m}\right\| \leq 1$ for each $m \in \mathbb{N}$.

Set $u=u_{1}+u_{2}$, where $u_{1}=P_{m} u$ and $u_{2}=\left(I-P_{m}\right) u$. We decompose Eq. (2.11) as follows:

$$
\frac{\partial u_{2}(t)}{\partial t}+2 \mu_{1} A u_{2}+B(u)-P_{m} B\left(u_{1}\right)+N(u)-P_{m} N\left(u_{1}\right)=f\left(t, u_{t}\right)-P_{m} f\left(t, u_{1 t}\right)+\left(I-P_{m}\right) g
$$

with initial data

$$
u_{2}(\tau+t)=\left(I-P_{m}\right) \phi(t), \quad t \in[-h, 0],
$$

and

$$
\frac{\partial u_{1}(t)}{\partial t}+2 \mu_{1} A u_{1}+P_{m} B\left(u_{1}\right)+P_{m} N\left(u_{1}\right)=P_{m} f\left(t, u_{1 t}\right)+P_{m} g
$$

with initial data

$$
u_{1}(\tau+t)=P_{m} \phi(t), \quad t \in[-h, 0] .
$$

We divide the proof into two steps:

Step 1: For every fixed $t \in \mathbb{R}$, any $B=\{B(t): t \in \mathbb{R}\} \in \mathcal{D}$ and any $\epsilon>0$ we observe that for any $T \geq t-s$ with $s \geq 0, U(T, t-s)(\phi)=\left\{u_{T}(\cdot ; t-s, \phi): u\right.$ is a strong solution to the problem (2.11) with $\phi \in B(t-s)$. We now show that condition (iii) of Proposition 2.6 holds. 
Taking the inner product of (4.17) with $A u_{2}=A\left(I-P_{m}\right) u$ in $H$, we have

$$
\begin{aligned}
& \frac{1}{2} \frac{d}{d t} a\left(u_{2}, u_{2}\right)+2 \mu_{1}\left(A u_{2}, A u_{2}\right)+\left\langle B(u)-P_{m} B\left(u_{1}\right), A u_{2}\right\rangle+\left\langle N(u)-P_{m} N\left(u_{1}\right), A u_{2}\right\rangle \\
& \quad=\left(f\left(t, u_{t}\right)-P_{m} f\left(t, u_{1 t}\right), A u_{2}\right)+\left(\left(I-P_{m}\right) g, A u_{2}\right) .
\end{aligned}
$$

Since $\left(u_{1}, u_{2}\right)=0$, from Hölder's inequality and Gagliardo-Nirenberg's inequality,

$$
\begin{aligned}
& \frac{1}{2} \frac{d}{d t} a\left(u_{2}, u_{2}\right)+2 \mu_{1}\left\|A u_{2}\right\|^{2} \leq\left|\left\langle B(u), A u_{2}\right\rangle\right|+\left|\left\langle N(u), A u_{2}\right\rangle\right|+\left|\left(f\left(t, u_{t}\right), A u_{2}\right)\right|+\left|\left(\left(I-P_{m}\right) g, A u_{2}\right)\right|, \\
&\left|\left(f\left(t, u_{t}\right), A u_{2}\right)\right| \leq \frac{\mu_{1}}{4}\left\|A u_{2}\right\|^{2}+\frac{L_{f}^{2}}{\mu_{1}}\left\|u_{t}\right\|_{C_{H}}^{2}, \\
&\left|\left(\left(I-P_{m}\right) g, A u_{2}\right)\right| \leq \frac{\mu_{1}}{4}\left\|A u_{2}\right\|^{2}+\frac{1}{\mu_{1}}\|g\|^{2}, \\
&\left|\left\langle B(u), A u_{2}\right\rangle\right| \leq\|B(u)\| \cdot\left\|A u_{2}\right\| \leq\|u\|_{L^{4}}\|\nabla u\|_{L^{4}}\left\|A u_{2}\right\| \leq c\|u\|^{\frac{1}{2}}\|\nabla u\|^{\frac{1}{2}}\|u\|^{\frac{1}{4}}\|\Delta u\|^{\frac{3}{4}}\left\|A u_{2}\right\| \\
& \leq c\|u\|^{\frac{3}{4}}\|\nabla u\|^{\frac{1}{2}}\|u\|_{W}^{\frac{3}{4}}\left\|A u_{2}\right\| \leq \frac{\mu_{1}}{4}\left\|A u_{2}\right\|^{2}+c\|u\|^{\frac{3}{2}}\|\nabla u\| \cdot\|u\|_{W}^{\frac{3}{2}} \\
& \leq \frac{\mu_{1}}{4}\left\|A u_{2}\right\|^{2}+c\|u\|^{\frac{3}{2}}\|u\|_{W}^{\frac{5}{2}}, \\
&\left|\left\langle N(u), A u_{2}\right\rangle\right|=\left|-\int_{\Omega}\{\nabla \cdot[\mu(u) e(u)]\} \cdot A u_{2} d x\right| \\
& \leq c(\|\nabla u\|+\|\Delta u\|) \cdot\left\|A u_{2}\right\| \leq \frac{\mu_{1}}{4}\left\|A u_{2}\right\|^{2}+c\|\Delta u\|^{2} .
\end{aligned}
$$

From (4.22)-(4.26) it follows

$$
\frac{d}{d t} a\left(u_{2}, u_{2}\right)+2 \mu_{1}\left\|A u_{2}\right\|^{2} \leq \frac{2 L_{f}^{2}}{\mu_{1}}\left\|u_{t}\right\|_{C_{H}}^{2}+\frac{2}{\mu_{1}}\|g\|^{2}+c\|u\|^{\frac{3}{2}}\|u\|_{W}^{\frac{5}{2}}+c\|u\|_{W}^{2} .
$$

On the other hand, from (4.15)-(4.16), we infer

$$
\left\|A u_{2}\right\|^{2} \geq \lambda_{m+1}\left(A u_{2}, u_{2}\right)=\lambda_{m+1} a\left(u_{2}, u_{2}\right),
$$

which along with (4.27) give

$$
\frac{d}{d t} a\left(u_{2}, u_{2}\right)+2 \mu_{1} \lambda_{m+1} a\left(u_{2}, u_{2}\right) \leq \frac{2 L_{f}^{2}}{\mu_{1}}\left\|u_{t}\right\|_{C_{H}}^{2}+\frac{2}{\mu_{1}}\|g\|^{2}+c\|u\|^{\frac{3}{2}}\|u\|_{W}^{\frac{5}{2}}+c\|u\|_{W}^{2} .
$$

Applying the Gronwall lemma to (4.28) in the interval $[\tau, t+\theta]$,

$$
\begin{aligned}
a\left(u_{2}(t+\theta), u_{2}(t+\theta)\right) \leq & a\left(u_{2}(\tau), u_{2}(\tau)\right) e^{-2 \mu_{1} \lambda_{m+1}(t+\theta-\tau)} \\
& +c \int_{\tau}^{t+\theta} e^{-2 \mu_{1} \lambda_{m+1}(t+\theta-s)}\left(\left\|u_{s}\right\|_{C_{H}}^{2}+\|g(s)\|^{2}+\|u(s)\|^{\frac{3}{2}}\|u(s)\|_{W}^{\frac{5}{2}}+c\|u(s)\|_{W}^{2}\right) d s .
\end{aligned}
$$

From (4.14) and Lemma 3.1 in [22], we can select $m+1$ large enough such that for all $\epsilon>0$ and $t \geq \tau+h$, we have $2 \mu_{1} \lambda_{m+1}-\sigma>0$, and

$$
\sup _{\theta \in[-h, 0]} \int_{\tau}^{t+\theta} e^{-2 \mu_{1} \lambda_{m+1}(t+\theta-s)}\|g(s)\|^{2} d s<\frac{c_{1} \epsilon}{2} .
$$


Thanks to Lemma 4.4 and 4.6, we can deduce that for large enough $m+1$,

$$
\begin{aligned}
& \sup _{\theta \in[-h, 0]} a\left(u_{2}(\tau), u_{2}(\tau)\right) e^{-2 \mu_{1} \lambda_{m+1}(t+\theta-\tau)} \\
& \leq c_{2} \sup _{\theta \in[-h, 0]}\left\|u_{2 \tau}\right\|_{C_{W}}^{2} e^{-2 \mu_{1} \lambda_{m+1}(t+\theta-\tau)} \leq \rho_{3}^{2}(\tau) e^{-2 \mu_{1} \lambda_{m+1}(t+\theta-\tau)} \leq \frac{c_{1} \epsilon}{4},
\end{aligned}
$$

and

$$
c \sup _{\theta \in[-h, 0]} \int_{\tau}^{t+\theta} e^{-2 \mu_{1} \lambda_{m+1}(t+\theta-s)}\left(\left\|u_{s}\right\|_{C_{H}}^{2}+\|u(s)\|^{\frac{3}{2}}\|u(s)\|_{W}^{\frac{5}{2}}+\|u(s)\|_{W}^{2}\right) d s<\frac{c_{1} \epsilon}{4} .
$$

Therefore, from (4.29)-(4.31) we have

$$
\left\|u_{2 t}\right\|_{C_{W}}^{2} \leq \frac{1}{c_{1}} a\left(u_{2}(t+\theta), u_{2}(t+\theta)\right)<\epsilon
$$

as claimed.

Step 2: We consider problem (4.19) and check condition (ii) in Proposition 2.6. Notice that

$$
\left\|A u_{1}\right\|_{W}^{2} \leq \lambda_{m}\left\|u_{1}\right\|_{W}^{2} \leq \lambda_{m}^{2}\left\|u_{1}\right\|^{2}
$$

Without loss of generality, we assume that $\theta_{1}, \theta_{2} \in[-h, 0]$ with $0<\theta_{1}-\theta_{2}<1$. Hence,

$$
\begin{aligned}
\left\|u_{1}\left(t+\theta_{1}\right)-u_{1}\left(t+\theta_{2}\right)\right\|_{W} & \leq \sqrt{\lambda_{m}}\left\|u_{1}\left(t+\theta_{1}\right)-u_{1}\left(t+\theta_{2}\right)\right\|=\sqrt{\lambda_{m}} \int_{t+\theta_{1}}^{t+\theta_{2}}\left\|\frac{d u_{1}}{d t}\right\| d t \\
& \leq \sqrt{\lambda_{m}} \int_{t+\theta_{1}}^{t+\theta_{2}}\left(2 \mu_{1} \sqrt{\lambda_{m}}\left\|u_{1}\right\|_{W}+\left\|B\left(u_{1}\right)\right\|+\left\|N\left(u_{1}\right)\right\|+\left\|f\left(s, u_{1 s}\right)\right\|+\left\|P_{m} g\right\|\right) d s .
\end{aligned}
$$

Since

$$
\begin{gathered}
\left\|B\left(u_{1}\right)\right\| \leq\left\|u_{1}\right\|_{L^{4}}\left\|\nabla u_{1}\right\|_{L^{4}} \leq c\left\|u_{1}\right\|^{\frac{1}{2}}\left\|\nabla u_{1}\right\|^{\frac{1}{2}}\left\|u_{1}\right\|^{\frac{1}{4}}\left\|\Delta u_{1}\right\|^{\frac{3}{4}} \leq c\left\|u_{1}\right\|^{\frac{3}{4}}\left\|\nabla u_{1}\right\|^{\frac{1}{2}}\left\|\Delta u_{1}\right\|^{\frac{3}{4}} \leq c\left\|\Delta u_{1}\right\|^{2}, \\
\left\|N\left(u_{1}\right)\right\| \leq c\left(\left\|\nabla u_{1}\right\|+\left\|\Delta u_{1}\right\|\right) \leq c\left\|\Delta u_{1}\right\|,
\end{gathered}
$$

and

$$
\left\|f\left(s, u_{1 s}\right)\right\| \leq L_{f}\left\|u_{1 s}\right\|_{C_{H}} .
$$

Thus, it follows from (4.32)-(4.35) that

$$
\left\|u_{1}\left(t+\theta_{1}\right)-u_{1}\left(t+\theta_{2}\right)\right\|_{W} \leq c \int_{t+\theta_{1}}^{t+\theta_{2}}\left(\left\|u_{1}(s)\right\|_{W}+\left\|u_{1}(s)\right\|_{W}^{2}+\left\|u_{s}\right\|_{C_{H}}+\left\|P_{m} g(s)\right\|\right) d s .
$$

Using Lemma 4.5 and Young's inequality,

$$
\begin{aligned}
c \int_{t+\theta_{1}}^{t+\theta_{2}}\left(\left\|u_{1}(s)\right\|_{W}+\left\|u_{1}(s)\right\|_{W}^{2}\right) d s & \leq c \int_{t+\theta_{1}}^{t+\theta_{2}}\left\|u_{1}(s)\right\|_{C_{W}}^{2} d s+c\left|\theta_{2}-\theta_{1}\right| \\
& \leq c \rho_{3}^{2}(t)\left|e^{-\sigma \theta_{1}}-e^{-\sigma \theta_{2}}\right|+c\left|\theta_{2}-\theta_{1}\right| .
\end{aligned}
$$


and

$$
c \int_{t+\theta_{1}}^{t+\theta_{2}}\left\|u_{s}\right\|_{C_{H}} d s \leq c \int_{t+\theta_{1}}^{t+\theta_{2}}\left\|u_{s}\right\|_{C_{H}}^{2} d s+c\left|\theta_{2}-\theta_{1}\right| \leq c \rho_{1}^{2}(t)\left|e^{-\sigma \theta_{1}}-e^{-\sigma \theta_{2}}\right|+c\left|\theta_{2}-\theta_{1}\right| .
$$

Noting that $g \in L_{l o c}^{2}(\mathbb{R} ; H)$,

$$
\begin{aligned}
\int_{t+\theta_{1}}^{t+\theta_{2}}\left\|P_{m} g(s)\right\| d s & \leq c \int_{t+\theta_{1}}^{t+\theta_{2}}\left(\left|\theta_{1}-\theta_{2}\right|^{\frac{1}{2}}\|g(s)\|^{2}+\frac{1}{4\left|\theta_{1}-\theta_{2}\right|^{\frac{1}{2}}}\right) d s \\
& \leq c\left|\theta_{1}-\theta_{2}\right|^{\frac{1}{2}} \int_{t}^{t+\theta_{1}-\theta_{2}}\|g(s)\|^{2} d s+\frac{1}{4}\left|\theta_{1}-\theta_{2}\right|^{\frac{1}{2}}
\end{aligned}
$$

From (4.36)-(4.39), we obtain

$$
\left\|u_{1}\left(t+\theta_{1}\right)-u_{1}\left(t+\theta_{2}\right)\right\|_{W}=\left\|P_{m}\left(u\left(t+\theta_{1}\right)-u\left(t+\theta_{2}\right)\right)\right\|_{W}<\epsilon
$$

for any $\theta_{1}, \theta_{2} \in[-h, 0]$ with $\left|\theta_{1}-\theta_{2}\right|<\delta$, so condition (ii) in Proposition 2.6 is proved. By Lemma 4.6, we know that condition (i) in Proposition 2.6 holds true. Hence, we can conclude by Proposition 2.6 that the process $\{U(t, \tau)\}$ is pullback $\mathcal{D}-\omega$-limit compact in $C_{W}$.

This completes the proof.

\subsection{Pullback $\mathcal{D}$-attractor}

We now state and prove the second main result of the work.

Theorem 4.9. Suppose that (H1) - (H3) and (4.14) hold. Then the process $\{U(t, \tau)\}$ associated to problem (1.1)-(1.4) has a unique pullback $\mathcal{D}$-attractor $\left\{\mathcal{A}_{\mathcal{D}}(t)\right\}_{t \in \mathbb{R}}$ in $C_{W}$.

Proof. By Lemma 4.6, we know that $\{U(t, \tau)\}$ has a pullback $\mathcal{D}$-absorbing set in $C_{W}$, while Lemma 4.8 shows that $\{U(t, \tau)\}$ is pullback $\mathcal{D}-\omega$-limit compact in $C_{W}$. Consequently, the proof can be completed immediately by Proposition 2.7 .

\section{Conclusion}

We have obtained some results concerning the asymptotic behavior of solutions to a 2Ddimensional incompressible non-Newtonian fluid with delay forcing term.

But, in our opinion, there is still much work to be done in this field. For example, it will be very meaningful to obtain some results on the finite (fractal or Hausdorff) dimensionality of the pullback attractor. Also, we could consider the regularity of the attractor as well as its internal structure for which it is important to study the existence of steady-state solutions and their stability properties. Also the interesting and important 3D-dimensional case is worth being considered. We plan to analyze all these topics in some forthcoming papers. 


\section{Appendix}

To make easier the readability of this paper, in this section we recall some lemmas that have been used in the present work.

The following key lemmas have been cited in Section 2 of [3] with appropriate references:

Lemma 6.1. If $u \in H_{0}^{1}(\Omega)$, then

$$
\|u\|_{L^{4}(\Omega)} \leq 2^{1 / 4}\|u\|_{L^{2}(\Omega)}^{1 / 2}\|\nabla u\|_{L^{2}(\Omega)}^{1 / 2} .
$$

Lemma 6.2. If $u \in W$, then there exists a positive constants $c_{0}$, depending only on $\Omega$, such that

$$
\|\nabla u\|_{L^{4}(\Omega)} \leq c_{0}\|u\|_{H^{1}(\Omega)}^{1 / 2}\|u\|_{H^{2}(\Omega)}^{1 / 2} .
$$

Lemma 6.3. There exist two positive constants $c_{1}$ and $c_{2}$ which depend only on $\Omega$ such that

$$
c_{1}\|u\|_{W}^{2} \leq a(u, u) \leq c_{2}\|u\|_{W}^{2}, \quad \forall u \in W .
$$

Lemma 6.4. (Gronwall's Lemma, see [[17], p. 9]) Let $x, y, \Psi$ be real continuous functions defined in $[a, b], y(t) \geq 0$ for $t \in[a, b]$. We suppose that on $[a, b]$ we have the inequality

$$
x(t) \leq \Psi(t)+\int_{a}^{t} y(s) x(s) d s .
$$

Then

$$
x(t) \leq \Psi(t)+\int_{a}^{t} y(s) \Psi(s) \exp \left[\int_{s}^{t} y(u) d u\right] d s .
$$

in $[a, b]$. Particularly, if $\Psi$ is differentiable, then from (6.1) if follows that

$$
x(t) \leq \Psi(a) \exp \left(\int_{a}^{t} y(u) d u\right)+\int_{a}^{t} \exp \left(\int_{s}^{t} y(u) d u\right) \Psi^{\prime}(s) d s,
$$

for all $t \in[a, b]$.

Lemma 6.5. (Uniform Gronwall's Lemma [20]) Let $t \in \mathbb{R}$ be given arbitrarily. Let $g$, $h$ and y be three positive locally integrable functions on $(-\infty, t]$ such that $y^{\prime}$ is locally integrable on $(-\infty, t]$, which satisfy that

$$
\frac{d y}{d t} \leq g y+h \text { for } s \leq t
$$

and

$$
\int_{t-1}^{t} g(s) d s \leq a_{1}, \int_{t-1}^{t} h(s) d s \leq a_{2}, \int_{t-1}^{t} y(s) d s \leq a_{3}, \quad \forall s \leq t,
$$

where $a_{1}, a_{2}$ and $a_{3}$ are positive constants. Then

$$
y(t) \leq\left(a_{2}+a_{3}\right) e^{a_{1}}, \quad \forall s \leq t .
$$

Acknowledgements. We would like to thank the referees and the handling editor for the helpful and interesting suggestions which allowed us to improve the presentation of our paper. 


\section{References}

[1] Hyeong-Ohk Bae. Existence, regularity, and decay rate of solutions of non-Newtonian flow. J. Math. Anal. Appl., 231(2):467-491, 1999.

[2] Hamid Bellout, Frederick Bloom, and Jindřich Nečas. Young measure-valued solutions for non-Newtonian incompressible fluids. Comm. Partial Differential Equations, 19(1112):1763-1803, 1994.

[3] Frederick Bloom and Wenge Hao. Regularization of a non-Newtonian system in an unbounded channel: existence and uniqueness of solutions. Nonlinear Anal., 44(3):281-309, 2001.

[4] Frederick Bloom and Wenge Hao. Regularization of a non-Newtonian system in an unbounded channel: existence of a maximal compact attractor. Nonlinear Anal., 43(6):743-766, 2001.

[5] T. Caraballo, G. Łukaszewicz, and J. Real. Pullback attractors for asymptotically compact non-autonomous dynamical systems. Nonlinear Anal., 64(3):484-498, 2006.

[6] T. Caraballo and A. M. Márquez-Durán. Existence, uniqueness and asymptotic behavior of solutions for a nonclassical diffusion equation with delay. Dyn. Partial Differ. Equ., 10(3):267-281, 2013.

[7] T. Caraballo and J. Real. Attractors for 2D-Navier-Stokes models with delays. J. Differential Equations, 205(2):271-297, 2004.

[8] Tomás Caraballo and Renato Colucci. Pullback attractor for a non-linear evolution equation in elasticity. Nonlinear Anal. Real World Appl., 15:80-88, 2014.

[9] Tomás Caraballo, Renato Colucci, and Xiaoying Han. Non-autonomous dynamics of a semiKolmogorov population model with periodic forcing. Nonlinear Anal. Real World Appl., 31:661-680, 2016.

[10] Tomás Caraballo, José A. Langa, and James C. Robinson. Attractors for differential equations with variable delays. J. Math. Anal. Appl., 260(2):421-438, 2001.

[11] Tomás Caraballo, Antonio M. Márquez-Durán, and Felipe Rivero. Well-posedness and asymptotic behavior of a nonclassical nonautonomous diffusion equation with delay. Internat. J. Bifur. Chaos Appl. Sci. Engrg., 25(14):1540021, 11, 2015.

[12] Renato Colucci and Gerardo R. Chacón. Dimension estimate for the global attractor of an evolution equation. Abstr. Appl. Anal., pages Art. ID 541426, 18, 2012. 
[13] Renato Colucci and Daniel Nuñez. Periodic orbits for a three-dimensional biological differential systems. Abstr. Appl. Anal., pages Art. ID 465183, 10, 2013.

[14] Julia García-Luengo, Pedro Marín-Rubio, and José Real. Pullback attractors in $V$ for nonautonomous 2D-Navier-Stokes equations and their tempered behaviour. J. Differential Equations, 252(8):4333-4356, 2012.

[15] Julia García-Luengo, Pedro Marín-Rubio, and José Real. Pullback attractors for 2D NavierStokes equations with delays and their regularity. Adv. Nonlinear Stud., 13(2):331-357, 2013.

[16] B. Guo, G. Lin, and Y. Shang. Non-Newtonian Fluids Dynamical Systems. National Defense Industry Press, in Chinese, 2006.

[17] A. Halanay. Differential equations: Stability, oscillations, time lags. Academic Press, New York-London, 1966.

[18] Jack K. Hale and Sjoerd M. Verduyn Lunel. Introduction to functional-differential equations, volume 99 of Applied Mathematical Sciences. Springer-Verlag, New York, 1993.

[19] Zhiyuan $\mathrm{Hu}$ and Yejuan Wang. Pullback attractors for a nonautonomous nonclassical diffusion equation with variable delay. J. Math. Phys., 53(7):072702, 17, 2012.

[20] Jae Ug Jeong and Jong Park. Pullback attractors for a $2 D$-non-autonomous incompressible non-Newtonian fluid with variable delays. Discrete Contin. Dyn. Syst. Ser. B, 21(8):2687$2702,2016$.

[21] O. Ladyzhenskaya. New equations for the description of the viscous incompressible fluids and solvability in the large of the boundary value problems for them, in: Boundary Value Problem of Mathematical Physics. American Mathematical Society, Providence, 1970.

[22] Songsong $\mathrm{Lu}$, Hongqing $\mathrm{Wu}$, and Chengkui Zhong. Attractors for nonautonomous 2D Navier-Stokes equations with normal external forces. Discrete Contin. Dyn. Syst., 13(3):701$719,2005$.

[23] Qingfeng Ma, Shouhong Wang, and Chengkui Zhong. Necessary and sufficient conditions for the existence of global attractors for semigroups and applications. Indiana Univ. Math. J., 51(6):1541-1559, 2002.

[24] J. Málek, J. Nečas, M. Rokyta, and M. Ružička. Weak and measure-valued solutions to evolutionary PDEs, volume 13 of Applied Mathematics and Mathematical Computation. Chapman \& Hall, London, 1996.

[25] Pedro Marín-Rubio, José Real, and José Valero. Pullback attractors for a two-dimensional Navier-Stokes model in an infinite delay case. Nonlinear Anal., 74(5):2012-2030, 2011. 
[26] James C. Robinson. Infinite-dimensional dynamical systems. Cambridge Texts in Applied Mathematics. Cambridge University Press, Cambridge, 2001. An introduction to dissipative parabolic PDEs and the theory of global attractors.

[27] Roger Temam. Infinite-dimensional dynamical systems in mechanics and physics, volume 68 of Applied Mathematical Sciences. Springer-Verlag, New York, second edition, 1997.

[28] Yejuan Wang and Peter E. Kloeden. The uniform attractor of a multi-valued process generated by reaction-diffusion delay equations on an unbounded domain. Discrete Contin. Dyn. Syst., 34(10):4343-4370, 2014.

[29] Caidi Zhao. Pullback asymptotic behavior of solutions for a non-autonomous non-Newtonian fluid on two-dimensional unbounded domains. J. Math. Phys., 53(12):122702, 22, 2012.

[30] Caidi Zhao. Dynamics of non-autonomous equations of non-Newtonian fluid on 2D unbounded domains. Dyn. Partial Differ. Equ., 10(3):283-312, 2013.

[31] Caidi Zhao and Yongsheng Li. $H^{2}$-compact attractor for a non-Newtonian system in twodimensional unbounded domains. Nonlinear Anal., 56(7):1091-1103, 2004.

[32] Caidi Zhao, Yongsheng Li, and Shengfan Zhou. Regularity of trajectory attractor and upper semicontinuity of global attractor for a 2D non-Newtonian fluid. J. Differential Equations, 247(8):2331-2363, 2009.

[33] Caidi Zhao, Guowei Liu, and Rong An. Global well-posedness and pullback attractors for an incompressible non-Newtonian fluid with infinite delays. Differ. Equ. Dyn. Syst., 25(1):3964, 2017.

[34] Caidi Zhao and Shengfan Zhou. Pullback attractors for a non-autonomous incompressible non-Newtonian fluid. J. Differential Equations, 238(2):394-425, 2007.

[35] Caidi Zhao, Shengfan Zhou, and Yongsheng Li. Trajectory attractor and global attractor for a two-dimensional incompressible non-Newtonian fluid. J. Math. Anal. Appl., 325(2):1350 1362, 2007.

[36] Kaixuan Zhu and Chunyou Sun. Pullback attractors for nonclassical diffusion equations with delays. J. Math. Phys., 56(9):092703, 20, 2015. 